\title{
LA DISCREPANCIA ENTRE LA INVESTIGACIÓN Y LA PRÁCTICA CLÍNICA DE LA TERAPIA DE CONDUCTA
}

\author{
ELISARDO BECOÑA
}

\author{
Universidad de Santiago de Compostela
}

(Recibido el 14 de abril de 1999)

\begin{abstract}
La terapia y modificación de conducta se ha consolidado como la terapia psicológica de mayor relevancia y eficacia en sus 40 años de existencia. Sin embargo cada vez más se hace evidente el surgimiento de discrepancias entre la investigación que sustenta sus técnicas de tratamiento y la práctica de la misma por parte de los psicólogos clínicos. Se analizan distintas discrepancias que se vienen arrastrado desde hace años (p. ej., el diagnóstico psiquiátrico; evaluación conductual vs. psicodiagnóstico clásico; casos simples vs. complejos; tratamientos simples vs. complejos; tratamiento fijo vs. cambiante; terapeutas en la investigación y en la práctica clínica; los diseños de caso único; criterio clínico vs. estadístico; poblaciones análogas vs. clínicas; duración de la terapia; éxitos y fracasos; y, seguimientos) así como la situación actual. Dentro de ésta se analiza el estado de las anteriores así como la escasa incidencia de la investigación en la práctica clínica, el paso del entrenamiento formal a la práctica clínica, el tiempo dedicado a la lectura (actualización) por parte de los clínicos e investigadores, la primacía de varios problemas clínicamente representativos, la eficacia en la investigación y práctica clínica y sus puntos fuertes y débiles. Se concluye que una parte importante de lo expuesto se relaciona con el paso de la teoría a la práctica y de la práctica a la tecnología y que ello es habitual en todas las ciencias aplicadas. El ser conscientes de ello puede ayudar a solventar algunas de las discrepancias que se han analizado.
\end{abstract}

Palabras clave: terapia de conducta, modificación de conducta, investigación, práctica clínica, discrepancias.

\section{Discrepancies between research and clinical practice of behavior therapy}

Behavior therapy and modification, with $\mathbf{4 0}$ years of history, it is today consolidated as the more relevant and efficcient of the psychological treatments. However it becomes evident the emergence of discrepancies among the investigation that sustain their treatment techniques and the practice of the same one on the part of the clinical psychologist. Different discrepancies are analyzed that are come crawling for years (e.g., the role of psychiatric diagnosis; behavioral assessment vs. traditional psychological assessment; single cases vs. complex cases; fixed treatment vs. changing treatment; therapist in the research and in the clinical practice; the designs of $\mathrm{N}=1$; the clinical significance vs. the statistical significance; analogue samples vs. clinical samples; duration of therapy; successes and failures; and follow-ups) as well as the current situation. We analized the state of the previous one as well as the little incidence of the research in the clinical practice, the step of the formal training to the clinical practice, the time dedicated to the reading on the part of the clinical and researchers, the primacy of several problems clinically representatives, the effectiveness in the research and in the clinical practice, and its strong and weaks points. We conclude that an important part of the above is related with the step from the theory to the practice and of the practice to the technology and this is usual in all the applied sciences. To know this can help it to solve some of the discrepancies that have been analyzed.

Key words: behavior therapy, behavior modification, research, clinical practice, discrepancies. 


\section{INTRODUCCIÓN}

Desde el surgimiento de la terapia y/o modificación de conducta a finales de los años 50 hasta el presente se ha recorrido un largo camino, tanto en su aspecto experimental, tecnológico como aplicado. En el momento actual las técnicas conductuales constituyen el bagaje más importante de que disponemos en psicología clínica, habiendo mostrando sus técnicas de tratamiento que no sólo son eficaces sino también que son eficientes y de coste razonable. El estar el tratamiento claramente estructurado, manualizado y evaluado ha ayudado en grado importante a esta consolidación. Sin embargo, desde poco después de su surgimiento comenzó a ocurrir lo que es característico a una ciencia aplicada: que no siempre hay una concordancia completa entre la investigación, altamente controlada y en condiciones óptimas, y la práctica de esa ciencia basada en la investigación. Estas discrepancias entre investigación y práctica clínica son las que analizamos a continuación.

En 1986 revisamos las discrepancias existentes entre la teoría e investigación y la práctica clínica de la modificación de conducta (Becoña, 1986). A pesar de que desde una perspectiva ajena a la modificación de conducta un observador podría decir de la misma que su metodología es muy refinada, que obtiene buenos resultados terapéuticos, que su rango de aplicación es amplio, etc., la realidad de los últimos años indica que hay fuertes discrepancias entre la teoría por una parte, la investigación por otra y la práctica clínica en el contexto real. Por suerte, este fenómeno, ha ido cambiando paulatinamente en los últimos años, en lo que atañe al distanciamiento entre una y otra parte de la modificación de conducta, aunque ello ha sido a costa de perder algunos de sus elementos más característicos (p. ej., los estudios basados princi- palmente en el laboratorio) y su necesidad de "socializarse" en el contexto social de la psicoterapia en el sentido más amplio. Esto tiene sus ventajas y desventajas, pero constituye también una prueba de madurez y de salto completo al mundo complejo de la clínica.

Para que se vea un poco como ha cambiado la situación en no más de una década, vamos a comparar las discrepancias que nosotros apuntamos en 1986 con las que hoy apreciamos, doce años después, en 1998, a las puertas del próximo siglo XXI.

\section{DISCREPANCIAS OBSERVADAS EN 1986}

Las discrepancias que nosotros considerábamos que existían en 1986 entre la teoría e investigación y la práctica clínica de la terapia de conducta eran doce (Becoña, 1986): el diagnóstico psiquiátrico; evaluación conductual vs. psicodiagnóstico clásico; casos simples vs. casos complejos; tratamientos simples vs. tratamientos complejos; tratamiento fijo vs. tratamiento cambiante; terapeutas en la investigación y en la práctica clínica; los diseños de caso único; criterio clínico vs. criterio estadístico; poblaciones análogas vs. poblaciones clínicas; duración de la terapia; éxitos y fracasos; y, seguimientos. A continuación vamos a comentar brevemente estas discrepancias para, posteriormente, ver la situación actual de éstas y otras discrepancias, en el sentido de cuales se han superado y otras nuevas que han surgido.

1) Diagnóstico psiquiátrico. Mientras que el modelo conductual ha rechazado clásicamente el diagnóstico psiquiátrico, lo criticó duramente cuando surgió la modificación de conducta y defiende la evaluación conductual (FernándezBallesteros, 1981), o el diagnóstico con- 
ductual (Kanfer y Saslow, 1965, 1969) o análisis funcional (Llavona, 1984), como un elemento central a la misma e incluso definitorio, en la práctica clínica frecuentemente los terapeutas de conducta han tomado prestados los conceptos más clásicos de la psicopatología (ej. neurosis) y, desde la aparición del DSM-III (American Psychiatric Association, 1980) relevantes autores conductuales (ej. Bellack y Hersen, 1985; Last y Hersen, 1985; Turner y Ascher, 1985), han defendido la utilización del diagnóstico psiquiátrico, como elemento de intercomunicación entre profesionales. Esto ha significado la incapacidad de extender un diagnóstico conductual - por ejemplo el desarrollado por Kanfer y Saslowque pueda ser utilizado en la práctica clínica cotidianamente. Esta discrepancia que nosotros apuntábamos ha sido superada en el sentido actual, dado que la psicología clínica en general, y la terapia de conducta en particular, hoy han asumido totalmente el diagnóstico psiquíatrico, véase el actual DSM-IV, como un elemento de intercomunicación entre profesionales, administraciones y pagadores de servicios, lo que ha constituído en la perspectiva histórica una revolución. Por contraposición esto ha llevado a darle un menor peso al análisis funcional, como analizaremos posteriormente, en el contexto clínico.

2) Evaluación conductual versus psicodianóstico clásico. La evaluación conductual surgió debido a las insuficiencias del psicodiagnóstico clásico (Nelson, 1983), siendo fuertemente criticado en los comienzos de la modificación de conducta. La modificación de conducta desarrolló desde sus inicios de modo muy importante todas las técnicas de evaluación conductual, con el objetivo de que a través de esas técnicas se pudiese realizar un adecuado análisis funcional. Los manuales sobre esta temática, tanto en aquellos años como ahora son abundantes (p. ej., los capítulos del libro de Labrador, Cruzado y Muñoz, 1993).

Sin embargo, hace unos 15 años se vivió una importante crisis sobre esta cuestión, como reflejaban varios autores cuando hablaban de que se vivía un periodo de "desilusión" (Nelson, 1983) por la evaluación conductual, y que además aparecía reflejado en diversas encuestas, como la de Piotrowski y Keller (1984), con miembros de la Association for Advancement of Behavior Therapy (AABT), donde los terapeutas de conducta utilizaban en ese momento frecuentemente medidas objetivas de personalidad, en menor grado medidas conductuales ( $p$. ej., mientras que el MMPI que lo utilizaban en un $70 \%$ de las veces, la observación sólo en un $20 \%$ y el análisis conductual en un $12 \%$ ), y llegaban incluso a utilizar alguna técnica proyectiva (p. ej., el TAT en el $38 \%$ de los casos).

Lo anterior venía a decir claramente que los teóricos e investigadores iban por un lado y los clínicos por otro. Mostraba además que las técnicas de evaluación conductual eran complejas, difíciles de implantar en las situaciones reales, caras en personal y tiempo, etc. El surgimiento del diagnóstico psiquiátrico más objetivo y centrado en los síntomas, a partir del DSM-III, ayudó a esta crisis, al tener un modo de "clasificar» más objetivo del que habia hasta ese momento.

Esto fue aprovechado además por un amplio grupo de personas que llevaban muchos años trabajando en la elaboración de tests o cuestionarios, surgiendo un nuevo "boom» de construcción, validación y utilidad de los test psicológicos. La realidad es que el diagnóstico clínico, siempre ha sido algo esencial para el psicólogo cuando tiene que analizar los problemas y dificultades que tiene la persona que acude en busca de ayuda profesional (Blanco, 1988). Este hecho se 
ha visto reflejado en las publicaciones, planteamiento de los casos, etc. Hoy la realidad nos dice que cualquier trabajo publicado exige un diagnóstico psiquiátrico claro (DSM-IV), su evaluación con un procedimiento de entrevista clínica estandarizada junto a otras pruebas adicionales que le podamos aplicar, al tiempo que no aparece la evaluación conductual o el análisis funcional por sitio alguno. De nuevo queda reducido al caso único, cuando así ocurre.

\section{3) Casos simples versus casos com-} plejos. Mientras que en las investigaciones clínicas se seleccionan a los sujetos con un trastorno único (simple) y preciso (p. ej., trastorno depresivo mayor), los sujetos que acuden normalmente a tratamiento suelen tener problemas complejos (Turner y Ascher, 1985). Mientras que a nivel investigador los casos complejos (p. ej., un sujeto que tiene al mismo tiempo un trastorno de ansiedad generalizada, depresión y graves problemas maritales) se rechazan, no ocurre esto en la clínica.

Sin embargo superar este problema es complejo en algunos niveles. La ciencia pretende la clasificación y organización adecuada de la realidad. Los casos simples posibilitan más fácilmente esta clasificación que los casos complejos. También, por cuestiones didácticas, es más fácil tener un número de categorías manejables y no otro que sea inmanejable. La investigación, finalmente, trata de controlar las variables del modo más adecuado para evitar los efectos de confusión. Todo esto unido lleva a que esta discrepancia haya existido y siga en parte existiendo, y no solo en la psicología, sino en muchas otras ciencias. Sí hay que decir que, en los últimos años, se han dado pasos importantes para solucionar estos problemas, aunque no siempre se reconoce que esto ocurre. Volveremos más adelante sobre este punto.
Lo que si es claro es que si tenemos una discrepancia entre la investigación, que selecciona casos simples en el sentido de que tengan un solo diagnóstico y los casos sean casi "puros» al no tener otros problemas graves o patologías asociadas, y en la clínica junto a los casos "puros" también aparecen aquellos «complejos» con más de un problema, nos vamos a encontrar con todo un conjunto de discrepancias a partir de ello, como tipo de tratamiento, terapeutas, seguimiento, etc.

4) Tratamiento simple versus tratamiento complejo. La existencia de casos simples en la investigación y casos complejos con frecuencia en la práctica clínica, nos conduce a que en la investigación se aplique un tratamiento, normalmente corto y claramente delimitado, mientras que en la clínica para los casos complejos se dediquen más sesiones y sea necesario utilizar más de una técnica simple o más de un paquete de tratamiento. Esto se refleja claramente en que los informes de investigación hablan habitualmente de 8 a 10 sesiones de tratamiento, mientras que en la práctica clínica del terapeuta de conducta puede en algunos casos llevar varias decenas de sesiones (Fishman y Lubetkin, 1983).

Hace 15 años comenzaron a oirse las primeras voces sobre este tema. Un claro ejemplo se podía ver en el libro de casos de Hersen y Last (1985), en donde, como hoy es mucho más claro, el número de sesiones, incluso para casos simples, dependía más del trastorno que de un promedio para cualquier trastorno. Intervenciones en problemas como obesidad, indican claramente que las típicas 8 a 10 sesiones a las que nos tiene acostumbrada la investigación para muchos trastornos, es insuficiente en ella. Más recientemente, esto ha cambiado en parte, dado que hay una importante variabilidad en el número de sesiones dependiendo de cada trastorno concreto. 
5) Tratamiento fijo versus tratamiento cambiante. En la línea de lo anterior, mientras en la investigación el tratamiento se lleva a cabo según un plan previamente preestablecido - y por tanto sin posibilidad de cambios en el trascurso del mismo-, en la práctica clínica en muchos casos es preciso y conveniente cambiar el plan de tratamiento que se estaba llevando a cabo. En el primer caso el objetivo es cumplir el plan establecido; en el segundo que un tratamiento sea eficaz en un determinado paciente.

La anterior cuestión no es posible solucionarla y hay que asumir que la investigación tiene un fin y un sentido: evaluar tratamientos para conseguir mejorar la eficacia de nuestras intervenciones. Ello exige unos controles metodológicos rigurosos, entre los que están que los sujetos sean equivalentes en su trastorno y en sus características demográficas, la asignación de los sujetos al azar a un grupo de tratamiento y control, la aplicación del tratamiento tal como está descrito (hoy, en forma de manual de tratamiento), la misma evaluación para todos, y un adecuado seguimiento para comprobar que los resultados se mantienen a corto, medio y largo plazo.

En la clínica sí que es cierto que lo que se pretende es obtener el mejor nivel de eficacia con un cliente concreto. Para ello, el clínico tiene que tener una buena formación previa, conocer qué es lo más eficaz en ese momento (a través de lo que lee y conoce sobre estudios de eficacia para distintos trastornos) y combinar su experiencia con todo lo anterior. Pero no son incompatibles un nivel y otro. Más bien el segundo nivel (la práctica clínica) es posible llevarla a cabo adecuadamente cuando tenemos bien evaluados los tratamientos para cada trastorno (investigación). Se precisa tener buenos investigadores y clínicos para el avance de los tratamientos psicológicos. Hacer ambas cosas facilita conocer cuál es el camino más adecuado para este avance.

6) Terapeutas en la investigación y en la práctica clínica. Mientras que los terapeutas que llevan a cabo los tratamientos en la investigación son normalmente recien licenciados o con pocos años de experiencia, los terapeutas de la práctica clínica tienen la mayoría de las veces más años de práctica y de experiencia (p. ej., Bergin y Garfield, 1994). Esta discrepancia siempre se ha apuntado y ha sido en ocasiones una fuente de crítica sobre los tratamientos psicológicos.

Una de las líneas de superación de este problema han sido los estudios multicéntricos sobre evaluación de distintas técnicas psicológicas, en comparación con otras farmacológicas, para distintos trastornos. Quizás el ejemplo más conocido y reciente es el estudio sobre la eficacia de distintos tratamientos para el tratamiento de la depresión, auspicado por el National Institute of Mental Health (ver Elkin, 1994). En éste se compararon la terapia cognitiva de Beck, la psicoterapia interpersonal, farmacoterapia (imipramina) y una condición placebo. Los terapeutas que llevaron a cabo cada una de las intervenciones, fuese psicológica o farmacológica, eran clínicos experimentados en cada uno de esos tratamientos, fueron a su vez entrenados concienzudamente, y todas sus intervenciones terapéuticas grabadas en video. Este es un ejemplo que permite solucionar el anterior problema.

7) Los diseños de caso único en la investigación y en la práctica clínica. Una de las característica definitorias de la modificación de conducta desde sus inicios fue el desarrollo de los diseños de $\mathrm{N}=1$. Partiendo de Skinner (1957), que defendió este tipo de diseños como el único adecuado, el reflejo bibliográfico, en forma de manuales, sobre los diseños 
de $\mathrm{N}=1$ o de caso único es abundante (p. ej., Arnau, 1984, Hersen y Barlow, 1984, Kazdin, 1994).

Sin embargo, cuando pasamos de la teoría a la práctica observamos que los diseños de $\mathrm{N}=1$ se utilizan fundamentalmente en la investigación y escasamente en la práctica clínica. Incluso es más, la mayoría de los diseños que aparecen en los manuales son imprácticos, difíciles de aplicar o hay muy pocos casos en la literatura con los mismos.

¿Por qué ocurre esto?, ¿por qué se utilizan tan poco? A pesar de que los diseños de $\mathrm{N}=1$ tienen una indudable utilidad, hay varias razones que explican su baja utilización, como son el gran control que exigen, problemas éticos o de establecer una línea base adecuada, complejidad de su análisis estadístico, coste, etc. (Becoña, 1986; Hayes, 1981; Wilson, 1982).

En la práctica clínica el objetivo básico es el tratamiento, conseguir que el cliente mejore en el menor tiempo posible. Va a depender del terapeuta el dedicar más o menos tiempo a la evaluación y al establecimiento de la línea base para la utilización o no de estos diseños. Sin embargo, creemos que este tipo de diseños es un modo adecuado de abordar muchos casos, especialmente aquellos sobre los que no hay un tratamiento claramente establecido, en aquellos en los que el terapeuta tiene poca experiencia terapéutica, o cuando quiere poner en marcha nuevas técnicas de tratamiento. Además, dentro de la metodología de investigación de un tratamiento psicologico, el estudio de un caso con un diseño $\mathrm{N}=1$ puede aportar mucha información tanto al clínico como a la ciencia psicológica (ver Maciá y Méndez, 1988).

8) Criterio clínico o terapéutico y criterio estadístico o psicométrico. Mientras que a nivel de investigación prima el criterio estadístico, en la clínica priva el cri- terio terapeutico o de mejora del sujeto tanto objetiva como subjetivamente.

El criterio clínico o terapéutico (Kazdin, 1982, 1992) se refiere a la significación clínica o aplicada de los cambios conductuales o si el tratamiento es eficaz a tenor de las diferencias que se puedan producir en el funcionamiento cotidiano del cliente. El criterio estadístico, experimental o psicométrico, se refiere al modo en que los datos son evaluados para determinar si el tratamiento tuvo algún efecto. Puede hacerse con los diseños de caso único, mediante inspección visual en el caso más simple o aplicando una prueba estadística, o también comparando un grupo de sujetos con otro. El propósito del criterio experimental es decidir si se ha demostrado un auténtico cambio y si este cambio puede ser atribuido al tratamiento.

La visión que tiene el clínico sobre ambos criterios queda expresada claramente en las siguientes palabras de Kazdin (1982) «los resultados de la investigación que se centran en los cambios estadísticamente significativos pueden no ser importantes; el clínico está interesado en producir un efecto clínicamente significativo, por ejemplo, un cambio que es claramente evidente en la vida diaria del paciente» (p. 14). Además, cuando el cambio clínico es evidente, el terapeuta lo aprecia, el cliente lo reconoce y una mera inspección visual de los datos (Eadington, 1984) nos permitiría ver ese cambio sin más, aunque sería también deseable y fácil de comprobar que el mismo también es estadísticamente significativo, aunque en la clínica es claro que la significación estadística está, o al menos debe estar, subordinada a la significación clínica.

Propuestas como las de Barlow, Hayes y Nelson (1984) en libros que han sido muy influyentes entre los clínicos, han llevado a que hoy en la investigación no siempre sea suficiente el criterio estadís- 
tico y haya que demostrar también que junto a la significación estadística ha habido una significación clínica. En España Páez, Echeburúa y Borda (1993) ha mostrado modos efectivos de evaluarlo desde un contexto clínico.

\section{9) Poblaciones análogas versus} poblaciones clínicas. Una discrepancia que, por suerte, hoy está casi totalmente superada, pero que hace 15 años todavía coleteaba, era la utilización frecuente de poblaciones análogas de trastornos clínicos en la investigación, mientras que en la clínica las poblaciones, como su misma palabra indica, eran poblaciones clínicas.

El terapeuta de conducta en su práctica clínica ha visto siempre sujetos con problemas reales y concretos, mientras que a lo largo de la historia de la investigación de muchos trastornos o problemas conductuales el investigador ha utilizado frecuentemente sujetos de poblaciones análogas (p. ej., estudiantes que han sido seleccionados para aplicarles dos técnicas de reducción de la ansiedad, en función de sus puntuaciones en una escala de ansiedad). Un ejemplo bien conocido ha sido el gran número de estudios que se han llevado a cabo con estudiantes universitarios con fobia a las serpientes en el pasado. Este era uno de los dos tipos fundamentales de investigaciones análogas que se han realizado (Bellack y Hersen, 1985). La primera es la ya comentada de utilizar poblaciones subclínicas, como estudiantes con fobia a las serpientes, y la segunda análogas de técnicas clínicas reales (p. ej., cuatro sesiones de desensibilización sistemática en grupo). Se utilizaban, por tanto, poblaciones sub-clínicas, que no poblaciones clínicas. Aunque la investigación análoga es un tipo específico de investigación (Bayés, 1984; Kazdin,1982, 1986), previa a una investigación con sujetos con ese problema clínico real, sólo debe utilizar- se en este contexto, a pesar de la dificultad que acarrea en algunas ocasiones conseguir sujetos clínicos para las investigaciones, por diversas razones.

Hoy esta discrepancia se ha superado, creemos, que totalmente. $\mathrm{Al}$ ser necesario tener un diagnóstico DSM-IV, CIE-10 o de una entrevista clínica estructurada derivada de los mismos, los casos que no tienen la denominación de "casos clínicos» quedan fuera de los estudios. Autores como Turner y Ascher (1985) ya afirmaban que muchos de los estudios análogos no aguantarían diagnósticos como el del DSM-III de aquel momento. Esto ha favorecido la utilización casi exclusiva de sujetos con trastornos clínicos y ha traído, como contrapartida y toque de atención, la revisión de muchas técnicas de modificación de conducta que se daban por bien asentadas y que en estos años se cuestionaron, especialmente algunas cognitivas, donde su evaluación era deficiente y controles meticulosos no siempre mostraron que eran eficaces (Becoña, 1991). Además, el surgimiento de la técnica del meta-análisis y la necesidad de reevaluar todas las investigaciones a través del mismo, ha producido otro de los cambios revolucionarios para la evaluación de los tratamientos psicológicos, a la que no es ajena la modificación de conducta, al tiempo que es la primera interesada, porque siempre ha defendido y defiende, la evaluación objetiva y científica de cualquier tratamiento psicológico.

10) Duración de la terapia en la investigación y en la práctica clínica. Los informes de investigación nos tienen acostumbrados a leer que las intervenciones terapéuticas en terapia de conducta son cortas, de 8 a 10 sesiones en la mayoría de los casos. Los libros de casos de terapia de conducta siguen una línea semejante, en donde con pocas sesiones, menos de una docena, el cliente/pacien- 
te supera su problema satisfactoriamente (p. ej., Maciá y Méndez, 1988). Sin embargo, en la práctica clínica habitual esto no ocurre exactamente así. Por una parte, si el caso es semejante a los descritos en la investigación (trastorno único, claramente definido y sin problemas concomitantes), el tratamiento en la clínica es rápido, como el que aparece en la investigación. Pero si el problema es más complejo, el número de sesiones en la clínica se va a incrementar de acuerdo a esa complejidad. $Y$, si tenemos que afrontar ciertos problemas, la duración del tratamiento va a ser muy superior a las 8-10 sesiones de que hablábamos, como tratamientos descritos de 18 meses para un problema de inadecuación social (Black y Schroeder, 1985), 11 meses para un problema de obesidad con 35 sesiones (Marcus, 1985), etc.

Hay otro factor importante relacionado con el número de sesiones de tratamiento: la creciente o mayor complejidad de los problemas de las personas que acuden a tratamiento. Ya en 1983 Fishman y Lubetkin (1983) apuntaban que el número medio de sus sesiones en la práctica privada con terapia de conducta se les había incrementado de 22 sesiones a principios de los años 70 a 50 en el momento de publicar el artículo (1983). Fishman y Lubetkin (1983) achacaban el aumento del número de las sesiones de terapia a que los problemas de los clientes se habian hecho menos concretos y mucho más complejos. La experiencia clínica en España indica que esto ocurre igualmente, tanto porque cada vez acuden más personas a tratamiento y con problemas en los que el psicólogo no intervenía hace 10 o 15 años, como porque las personas acuden cuando tienen un problema complejo o vienen en un estado de deterioro importante y no en las fases iniciales del mismo.

Lo que sí nos interesa destacar aquí, como ya hemos indicado (Becoña, 1986), es que no toda la terapia de conducta tenía una visión tan optimista del número de sesiones. Lazarus (1971) presentaba el tratamiento de una mujer con agorafobia que duró 38 sesiones a lo largo de cinco meses. Nótese que en esa época, incluso ese periodo de tiempo era muy reducido respecto a la psicoterapia tradicional que echaría dos o tres años para el mismo problema. Pero donde más claramente aparece la duración de la terapia de conducta es en uno de sus padres fundadores, Wolpe. Wolpe (1958) en su libro de Psicoterapia por inhibición recíproca presenta en una tabla de su libro (Wolpe 1958/1878, pp. 242-249) la duración del tratamiento en meses y el número de sesiones. Las ocho o diez sesiones a las que estamos habituados, solo constituía el $15.9 \%$ de los casos tratados por Wolpe, aunque el $26 \%$ de ellos eran tratados en un periodo de tiempo menor a dos meses. En aquellos casos graves, rebeldes o difíciles era más normal echar varios años en tratamiento, como es bien representativo el caso que estuvo siete años en tratamiento, con 426 sesiones, con las características clínicas de ansiedad interpersonal, fobias múltiples y estado obsesivo grave. Nótese que el libro de Wolpe, el clásico libro de Wolpe para la modificación de conducta, es un ejemplo que se pone siempre de lo corta que era la desensibilización sistemática, como realmente lo es de modo general, y mucho más rápida que otros tratamientos, pero no universalmente rápida.

11) Exitos y fracasos. No cabe duda de que la terapia de conducta ha aportado un conjunto de técnicas que han mostrado experimentalmente su eficacia en un considerable número de trastornos, siendo además sus profesionales numerosos y su produción científica extensa. Pero no siempre es eficaz. Por ello es relevante analizar por qué las técnicas conductuales no son efectivas en todos los casos. 
Wolpe (1958) hablaba de un porcentaje de éxito de aproximadamente un $90 \%$, porcentaje que se ha visto rebajada a un $70 \%$ en la encuesta de Swan y MacDonald (1978), aunque hoy sería un atrevimiento hablar de un porcentaje de éxito para la "terapia de conducta» para «todos» los «trastornos clínicos». Hoy los porcentajes de éxito hay que considerarlos para cada trastorno específico y en ellos ver el mejor o peor funcionamiento de las distintas técnicas conductuales, en donde en unos casos funcionan muy bien (p. ej., disfunciones sexuales) y en otras no tan bien (p. ej., trastornos de personalidad), como analizamos en un apartado posterior sobre la eficacia de la terapia de conducta.

Por lo que atañe al análisis de los fracasos en modificación de conducta, ha sido el libro de Foa y Emmelkamp (1983) el que más crudamente ha planteado este tema y en los diversos capítulos del mismo, escritos por relevantes terapeutas conductuales. Las razones que se han apuntado para que con anterioridad no se haya hablado más de este tema han sido para Emmelkamp y Foa (1983) el no aceptar las revistas artículos donde aparecen resultados negativos; el sentirse los autores forzados $\mathrm{y}$ casi obligados a demostrar la eficacia y no el cuestionamiento de sus técnicas, por las críticas que en otra época la terapia de conducta realizó a la psicoterapia tradicional; y, porque los investigadores, por múltiples razones bien comprensibles, prefieren indicar sus éxitos más que relatar sus fracasos.

Emmelkamp y Foa (1983), de modo genérico, clasifican en cuatro los fracasos que suelen tener los tratamientos conductuales. El primero son las negativas. Esto se refiere a que hay clientes que siendo apropiados para el tratamiento lo rehusan. Por tanto no llegan ni a empezarlo. El segundo son las retiradas. Hay clientes que no llegan a completar el tra- tamiento, retirándose del mismo en su trascurso. El terapeuta los clasifica en este caso como fracaso. El tercero atañe al cliente no respondiente, donde aunque se le aplica al sujeto el tratamiento (que por el terapeuta es considerado el adecuado para el mismo) no le resulta efectivo, no alcanzando el nivel de mejora que se esperaba antes del tratamiento. Sería el caso de fracaso por excelencia. El cuarto se refiere a las recaídas, el cliente logra el objetivo terapéutico en el tratamiento, pero con posterioridad no se mantiene y recáe. Este es un fenómeno típico de las conductas adictivas (Becoña, Lista y Froján, 1988).

Analizando más específicamente por qué se fracasa, Emmelkamp y Foa (1983) apuntan como fundamentales las siguientes: a) error en el diagnóstico; b) aplicación inadecuada del tratamiento; y, c) variables relacionadas con el mantenimiento de la ganancia (o recaída) a largo plazo pueden no ser las mismas que las existentes durante el curso de la terapia.

Los fracasos, como Emmelkamp y Foa (1983) afirman, no deben ser una fuente de desánimo, sino más bien todo lo contrario. Pueden ser, precisamente, una fuente invalorable de información que puede permitirnos conocer mejor los mecanismos subyacentes a nuestros tratamientos, y con ello poder perfeccionarlos y proponiendo otros nuevos. En el tratamiento de las conductas adictivas este tema, por ejemplo, tiene actualmente una gran relevancia, como es buen reflejo la prevención de la recaída (Marlatt y Gordon, 1985).

Por tanto, tanto en la investigación como en la clínica nos vamos a encontrar con fracasos. Más que una fuente de frustración tiene que ser un elemento que nos haga reconocer las limitaciones de nuestras técnicas de tratamiento, y una vez reconocido ésto, perfeccionarlas cada día para incrementar su eficacia. 
12) Seguimiento en la investigación y en la práctica clínica. La objetividad propugnada por la modificación de conducta y la evaluación empírica de sus resultados no siempre se ha visto reflejada en los necesarios seguimientos que son precisos para comprobar que las mejoras obtenidas en el tratamiento se mantienen a lo largo del tiempo, especialmente en la práctica clínica. En este tema se encuentra que, en la investigación, en contraposición a las escasas sesiones de tratamiento, los seguimientos suelen ser largos (de 6 a 12 meses), mientras que ocurre lo contrario en la práctica clínica: el número de sesiones es mayor y los seguimientos normalmente son inexistentes, por diversos motivos, tales como asunción del mantenimiento de la mejoría, el cliente no acude a la cita de seguimiento por encontrarse bien, el terapeuta tiene que asignarse un tiempo de su agenda y sus nuevos casos se lo impiden, etc. El teléfono o la carta es una posible solución, aunque debido a todas las razones hasta aquí expuestas ello es más propio del investigador que del clínico que supera día a día los problemas que se le plantean y evita otros que le roban tiempo innecesario. Quizás no es realista (Turner y Ascher, 1985) pensar que el clínico va a hacer todos los seguimientos o seguimientos largos. Con todo, creemos que llevar a cabo seguimientos es importante, tanto para que el propio terapeuta evalúe la eficacia de sus intervenciones como de la modificación de conducta en general (Kaplan, 1986). Esta era y es una limitación dentro de la discrepancia entre la investigación y la práctica clínica de la terapia de conducta.

Todas las discrepancias anteriores, aparecen claramente reflejadas en lo apuntado hace una década por Bellack y Hersen (1985), quienes llegaban a afirmar que había incluso personas que pensaban que existían dos clases de terapia de conducta. En la primera de ellas, la que se describe en los libros de texto, «el sujeto o cliente pide ayuda con un problema simple claramente definido, donde la naturaleza del mismo es tan obvia que podría ponérsele el título en la frente. Aquí no es preciso tomar decisiones de tratamiento, y el terapeuta es capaz de llevar a cabo todos los tratamientos exactamente como se especifican. Los clientes obedientemente acuden a todas las sesiones y son cooperativos a lo largo de las mismas. Nunca preguntan cuestiones sobre asuntos prosaicos como su imposibilidad de pagar los honorarios o el hecho de que el tratamiento no parece estar dirigido a los problemas reales. Nunca ocurre el que se descubra a mitad de tratamiento algún hecho que cambie el diagnóstico y la orientación del tratamiento. Los clientes no mejoran todos en esta terapia de conducta, pero el tratamiento no suele pasar de ocho a diez sesiones, sin hacer caso del resultado o de la variabilidad individual" (pp. 3-4). Por contra, en la otra terapia de conducta, la que se practica en clínicas, hospitales y consultas privadas "ésta "raramente" parece ser tan simple y directa como el primer tipo. Los clientes o pacientes se presentan con múltiples problemas que son generalmente difíciles de separar y categorizar. Sin embargo ellos frecuentemente tienen el hábito molesto de saltarse los planes de tratamiento no revelando nueva información hasta que están más cómodos con el terapeuta o por tener crisis que requieren su inmediata atención. No acuden siempre a las sesiones semanales, ni siguen las instrucciones, ni cooperan. Por su parte, el terapeuta en esta terapia de conducta siente la necesidad de ser sensible a los caprichos de sus clientes o pacientes. Además, frecuentemente disfruta hablando con ellos más que llevando a cabo tareas repetitivas altamente estructuradas. Consecuentemente, el tratamiento para cada cliente es individualizado y difícil de describir del 
modo objetivo preferido en el primer tipo de terapia de conducta. Es innecesario decir que este tipo de terapia de conducta típicamente requiere mucho más que las ocho o diez sesiones del primer tipo» (p. 4).

\section{SITUACIÓN ACTUAL: SUPERACIÓN DE ALGUNAS DISCREPANCIAS Y SURGIMIENTO DE OTRAS NUEVAS}

\section{Análisis de las anteriores discrepancias en el momento presente}

Algunas de las discrepancias anteriores hoy se pueden considerar resueltas 0 totalmente resueltas, mientras que otras siguen en el mismo estado. También, como veremos posteriormente, mientras que se resuelven algunas surgen otras nuevas.

Dentro de la evaluación la situación ha cambiado radicalmente en los últimos años. Mientras que el surgimiento del DSM-III (American Psychiatric Assocation, 1980), con un planteamiento innovador y más adecuado a los tiempos, no permitía claramente preveer su aceptación completa en el campo de la salud mental, después del DSM-III-R (American Psychiatric Association) y más recientemente del DSM-IV (American Psychiatric Association, 1994) era evidente que se iba a implantar. Desde el campo de la investigación conductual su aceptación ha sido completa en estos últimos años, precisamente por lo que se propugna como elemento básico del mismo: un sistema de comunicación entre los profesionales de la salud mental, para tener un lenguaje común para entenderse en un tema tan importante como es el diagnóstico (Frances, First y Pincus, 1997). Igualmente, la participación de relevantes figuras del campo conductual en los comités de expertos de distintos trastornos (p. ej., Barlow en el DSM-IV), ha facilitado aún más su aceptación. Y, el elemento básico de su aceptación en la investigación es la obligación de presentar en los artículos para su consideración para su publicación los casos de un trastorno con un diagnóstico aceptado internacionalmente, como es fundamentalmente el DSM-IV.

Desde la clínica, la penetración del diagnóstico psiquiátrico ha sido importante, aunque no exento de críticas y de propuestas alternativas a principios de los años 80 (ver Hersen, 1992). Quién se ha resentido de todo ello ha sido la evaluación conductual y el diseño de un tratamiento específico para cada individuo. Aunque esto es necesario en muchos casos, la utilización de los tratamientos estándares en los últimos años, ha ido en contra de esto. Al mismo tiempo, el psicodianóstico clásico ha sufrido una amplia transformación, habiéndose elaborado nuevos instrumentos e integrándose éstos en la propia evaluación conductual (Taylor, 1999). Queda más malparado el análisis funcional de la conducta.

No se ha resuelto el tema, porque no es resoluble tal como se realizan los tratamientos en la clínica y en la investigación sobre el tipo de casos y su abordaje según sean simples o complejos. En la clínica los casos siguen siendo en una parte de ellos complejos. En cambio en la investigación, aquellos casos que tienen más que el diagnóstico sobre el que interesa hacer el estudio, son excluidos del estudio, a través de la utilización de criterios de inclusión y criterios de exclusión. Esto que es bueno para la ciencia, no es tan bueno para los clínicos.

Sí ha cambiado de modo importante la cuestión de los terapeutas que aplican un tratamiento. Más que hablar hoy de si un terapeuta tiene mucha formación o poca formación, si su ojo clínico (subjetivamente) es mejor o peor, hoy lo importante es que el tratamiento se aplique según 
el programa propuesto. De ahí, la relevancia que hoy tiene la manualización de los tratamientos (p. ej., el libro de Van Hasselt y Hersen, 1996) y la comprobación de que el terapeuta aplica el tratamiento tal como ha sido diseñado y tal como consta en el manual del tratamiento. Esta línea parece fructífera y muestra que lo importante es el entrenamiento en la técnica específica, un buen entrenamiento, más que mucha práctica pero no siempre necesariamente guiada por un buen entrenamiento o una buena aplicación del mismo.

Acerca de los diseños de caso único se aprecia una disminución de su uso. La publicación de manuales sobre esta temática se ha reducido drásticamente en los últimos años, como de artículos sobre aspectos metodológicos de los mismos, y son pocos los casos clínicos que se publican con diseños específicos de caso único. Unicamente queda vigente el diseño básico A-B, o de línea base y posterior aplicación del tratamiento, junto a llevar a cabo el posterior seguimiento (p. ej., Maciá y Méndez, 1988), lo cual consideramos negativo.

Relacionado con la irrupción del DSMIII y posteriores, y lo que ello implicaba, pronto desaparecieron los estudios análogos de problemas clínicos. Hoy es difícil encontrar un estudio de este tipo, con la excepción de los estudios básicos. Esto ha favorecido el que en los últimos años tengamos una gran cantidad de información sobre distintos trastornos clínicos al dejarse de hacer estudios con análogos, junto a seguirse incrementando exponencialmente las personas que trabajan en el campo de la psicología en el mundo y que hacen aportaciones clínicas diariamente.

Sobre la duración de la terapia, los datos hay que analizarlos hoy por trastorno más que por una media entre los distintos trastornos. No contamos realmente con muchos datos sobre esta cues- tión, aunque es un tema de preocupación. La duración de la terapia se asocia con el coste de la misma. Distintos análisis de esta temática nos han aportado datos importantes sobre qué terapia podemos elegir, en el alcoholismo, la ansiedad, la depresión, etc. En los próximos años éste es un aspecto que se va a potenciar por la necesidad de evaluar el coste-efectividad, y en ello tiene gran relevancia el número de sesiones.

Cualquier procedimiento terapéutico pretende obtener un buen nivel de eficacia. Los fracasos nunca se ven bien y se procura evitarlos tanto con los tratamientos que se aplican, con estrategias de prevención de la recaída o introduciendo otros tratamientos nuevos que nos puedan ayudar a mejorar nuestra eficacia. Por ello, éste es un tema de preocupación permanente y el análisis de las variables predictivas va en esta línea.

Relacionado en parte con lo anterior, hoy la investigación nos permite conocer cuando un seguimiento es necesario en un tratamiento, porque hay una alta probabilidad de recaída en los estudios realizados o es poco probable que ocurra. De este modo se ha solventado en parte la discrepancia aunque no totalmente. Esto es, si la probabilidad de recaída es importante, se incluyen sesiones de mantenimiento a largo plazo; si la probabilidad es menor se programan sesiones de mantenimiento o seguimiento más espaciadas; si la probabilidad de recaída es baja o casi inexistente se deja de ver a la persona una vez que supera su problema. De este modo el clínico encuentra en la investigación una aportación importante para su toma de decisiones. La cronificación de muchos trastornos y la dificultad de otros, también ha facilitado el que éstos hagan más controles de seguimiento que en el pasado, ya que ello también facilita mantener más adecuadamente las ganancias obtenidas en el tratamiento. 


\section{OTRAS NUEVAS DISCREPANCIAS O DIFERENCIAS ENTRE INVESTIGACIÓN Y PRÁCTICA CLÍNICA}

En estos últimos años se han solventado distintas discrepancias que se observaban hace 10 o más años y, al tiempo, han surgido otras nuevas o claras diferencias entre la investigación y la práctica clínica en terapia y modificación de conducta. A continuación vamos a indicar aquellas que en nuestra consideración nos parecen más relevantes: la escasa incidencia de la investigación en la práctica clínica; la falta de difusión de procedimentos efectivos de la investigación a la práctica clínica; del entrenamiento formal a la práctica clínica; las lecturas de unos y de otros: los investigadores en exceso, los clínicos en defecto; la primacía de varios problemas clínicamente representativos en la práctica clínica; la eficacia de la intervención clínica en la investigación y práctica clínica; puntos fuertes y débiles de la terapia de conducta, junto a un apartado posterior que dedicamos a la relación entre teoría y práctica y práctica y tecnología.

La escasa incidencia de la investigación en la práctica clínica

Lo que diferencia a la modificación de conducta de otras formas de tratamiento psicológico es que su práctica está basada en la ciencia, en los resultados de la investigación científica. Sin embargo, hoy no es del todo claro que ello sea siempre así. En Estados Unidos, se ha optado en Psicología por un modelo de formación científico-práctico, a partir de la conferencia de Boulder en 1949 (Raimy, 1950). Sin embargo, desde ese momento, en ocasiones distintos autores han insistido en que ello no se estaba consiguiendo. En 1981, David Barlow
(1981) afirmaba que "en el presente, la investigación clínica tiene poca o ninguna influencia sobre la práctica clínica» (p. 147). En 1993, Cyril Franks (1993) insistía en que la ciencia y la práctica clínica debían ir de la mano, aunque había que mejorar esa relación. El artículo de Sobell (1996) sobre esta temática, indica que la integración todavía no ha ocurrido, siendo una asignatura pendiente. En España, aunque tenemos pocos datos sobre ello, la situación es semejante ( $p$. ej., la formación por el sistema P.I.R.).

Por su parte, como afirma Lazarus (1994), los clínicos creen que el conocimiento les va a venir dado de sus observaciones clínicas -aunque sean sesgadas- y no de lo que se hace en la investigación. Esto ya fue apuntado por Miller (1987), quién refiriéndose a los tratamientos del alcoholismo, indicaba que demostrar que nuestras técnicas funcionan no es suficiente; hay que hacerselas llegar a los clínicos y convencerlos de que funcionan, dado que en éste como en otros campos, con frecuencia los clínicos ignoran las publicaciones útiles y relevantes. Además, la mayoría de las revistas científicas son inaccesibles a los clinicos y cuando las leen les parece difícil de leer, con estadísticos para ellos incomprensibles y que hace que no sean fáciles de manejar. Otros autores (p. ej., Goldfried, 1983) han insistido también en que los psicólogos clínicos suelen ignorar lo que se publica en las revistas científicas de su campo.

Sobell (1996) dice que es necesaria la realización de investigaciones rigurosas, bien controladas. Pero que una vez que ello se ha hecho es necesario que se difundan. Si esto no se hace, de poco sirve ese producto tan bueno (Barlow, 1994). Por ello Sobell (1996) sugiere que los investigadores tienen que aprender a escribir para los clínicos. Dos ejemplos recientes van en esta línea, las nuevas revistas Cognitive and Behavioral Practi- 
ce de la AABT e IN Session, publicada por Wiley. Además, si creemos que nuestras técnicas de intervención son clínicamente relevantes, debemos difundirlas como si de un producto empresarial se tratase. Esto exige estar cerca del consumidor, nuestros clientes en este caso, adecuarse a sus necesidades y, lo que casi nunca se ha hecho, desarrollarlo tomando ellos parte desde las primeras fases.

Otro problema relacionado con esto es que a nivel universitario, en Estados Unidos como en modo semejante en España, cada vez se prima más la investigación básica. La investigación aplicada se relaciona con la tecnología y se relega a un estatus académico secundario (Howard, 1998). La investigación del mundo real cada vez se hace más en hospitales, clínicas especializadas, institutos de investigación clínica, etc., por lo que cada vez se va desvinculando más del mundo académico. Es cierto que la investigación básica tiene poca relevancia para las situaciones aplicadas (Follette, 1998), porque la vida real es compleja y no se parece en nada a la situación del laboratorio. Mientras que para el clínico la investigación básica está fuera del mundo real (o inmediato), la investigación que se realiza en centros especializados, aunque centrada en el mundo real, no siempre se transmite adecuadamente sus resultados a sus destinatarios. Creemos, con todo, que la ciencia básica y la ciencia aplicada se pueden beneficiar mútuamente, permitiendo el desarrollo de tratamientos más eficaces (p. ej., Bootzin y McKinght, 1998).

La falta de difusión de procedimientos efectivos de la investigación en la práctica clínica

La difusión no es un proceso fácil. Hay casos históricos muy curiosos donde desde el descubrimiento de un hecho impor- tante hasta su implantación han pasado décadas o siglos (Sobell, 1996). Para Rogers (1995) los cuatro elementos esenciales del proceso de difusión son: innovación, canales de comunicación, tiempo y sistema social. Aquello que queremos difundir tiene que ser visto como nuevo, como una innovación; los canales de comunicación deben estar abiertos entre el que comunica y el que recibe; el tiempo atañe a que hay que tener expectativas realistas sobre la implantación (primero suele haber innovadores que empiezan a utilizar el nuevo procedimiento, antes de pasar a su posterior generalización, si es el caso); no hay que olvidarse del sistema social, especialmente de la opinión de los líderes y de los agentes de cambio sobre esa nueva innovación. A su vez, los factores que se ha encontrado que favorecen la adopción de una innovación son: ventaja real, compatibilidad, complejidad, comparabilidad y observabilidad (Rogers, 1995).

Sobell (1996) se hace una pregunta crucial sobre muchos tratamientos psicológicos probados eficazmente para distintos problemas pero que luego no se implantan, « ¿por qué nuestra investigación es ignorada?». Concretamente, esto es muy representativo en el campo del alcohol. Así, McCrady (1986) ha llegado a escribir algo tan elocuente como esto: "nuestro trabajo de investigación es impecable; nuestras aproximaciones al tratamiento son tan creativas y efectivas como cualquier otra aproximación; nuestro deesarrollo teórico cuidadoso y bien pensado. Desafortunadamente, nuestro trabajo es también invisible" (p. 173174). Esto es muy apreciable en el campo del alcohol, como puede verse en la extensa y comprensiva revisión de Miller et al. (1995). Lo eficaz no tiene porque ser lo más utilizado o lo más aceptado. La difusión y el marketing a veces modulan el resultado final. Claramente, los científicos no son buenos empresarios. 
Una de las aplicaciones exitosas, siguiendo una buena estrategia de difusión de programas puede verse en Sobell (1996) en la provincia de Ontario en Canadá, en la difusión de programas de alcoholismo. En su caso incluyeron a diversos miembros de la comunidad en todo el proceso de implantación del programa. Este es uno de los elementos esenciales para conseguir buenos resultados, al adecuarse a las necesidades de los clientes, proporcionar apoyo clínico a los terapeutas que lo están implantando con un buen feedback, hacerles buenos materiales clínicos que tengan disponibles, etc.

Otra cuestión relacionada con la difusión, es la necesidad de educar al público sobre el valor de la terapia de conducta, dado que la población todavia sigue teniendo ideas erróneas sobre la misma (Zeiss, 1997). Una buena ocasión es el desarrollo de las guías prácticas tanto dentro del campo de la psicología, como ha hecho el Task Force on Promotion and Dissemination on Psychological Procedures (1993), como se ve en las guías clínicas elaboradas por la Sociedad Americana de Psiquiatría, en donde sale muy bien parada la terapia de conducta o es la que viene a salir bien parada de todas las terapias psicologicas (p. ej., American Psychiatric Association, 1996), lo que al mismo tiempo está produciendo una fuerte reacción por parte del campo de la psicoterapia (p. ej., Garfield, 1996) aunque la evidencia de lo que funciona es claro. El markéting de lo que hacemos es del todo necesario (Arndorfer, Allen y Aljazirech, 1999).

Cateutelli, Rosenwasser y Skinner (1996) indican como la tecnología basada en la psicología del aprendizaje apareció con gran fuerza y optimismo, mostraron su efectividad en un amplio rango de problemas, pero no han llegado al nivel que hace unas décadas se creía. El problema de la difusión de los procedimientos ha sido una causa de ello.
También el problema de la imagen es importante. La tecnología conductual sufrio fuertes críticas desde sus inicios no ya dentro de la psicología, sino en ocasiones desde fuera de ella. Algunas de las polémicas, como la de Chomsky con Skinner sobre el lenguaje, creemos que tuvieron un gran eco social; lo mismo podemos decir de la polémica entre el psicoanálisis y la terapia de conducta. Estas polémicas no siempre se han resuelto en el campo de la academia o en el campo científico, sino que muchas veces se han ido hacia afuera, hacia los medios de comunicación, los círculos intelectuales, políticos, etc. Quizás el ejemplo más claro del problema de la difusión, en esta línea, es el de las técnicas conductuales en el alcoholismo. Estas chocan con el modelo social imperante de enfermedad (Cautelli et al., 1996; Sobell, 1996), llegando incluso los pacientes a tener una visión negativa de las propias técnicas conductuales por no encajar con el modelo de su "enfermedad». Como bien ha indicado Mandler (1995), la ciencia y las teorías crecen en un medio social propio, sino no les es posible aparecer y desarrollarse.

Las siguientes palabras de Sobell (1996) resumen lo que hay que hacer en el futuro: «la difusión, como un valor, deberá ser adoptado y ser un objetivo central en los próximos años» (p. 318), dado que "si nosostros estamos convencidos de que nuestros métodos consiguen una mejoría clínica significativa sobre otras prácticas alternativas, entonces tenemos la obligación de difundir dichos métodos» (p. 318).

\section{Del entrenamiento formal a la práctica clínica}

Un elemento que media parte de lo que estamos analizando es la relación que existe entre el entrenamiento formal de 
un clínico en su periodo de formación y lo que hace luego en su práctica clínica. A partir del modelo de Boulder norteamericano de hace ya casi 50 años se ha optado en todos los países por una formación del psicólogo clínico como científico y como clínico. La realidad no siempre indica que ello haya sido siempre bueno (ver Hayes, Follete, Dawes y Graves, 1995). Además, en los últimos años han surgido voces críticas buscando una solución a la discrepancia que se aprecia entre la formación, cierto tipo de formación, y los problemas a los que luego se tienen que enfrentar en su práctica clínica.

Cone, Alexander, Lichtszajn y Mason (1996) afirman que los programas de entrenamiento de terapia de conducta tienen que abandonar su «torre de cristal» y pasar a relacionarse con el mundo real, tal como la atención primaria, los sistemas de cuidado de salud, la empresa y la industria. Estos autores proponen un sistema más flexible de entrenamiento, donde se le da más énfasis a la experiencia de campo como un elemento básico del entrenamiento, saliendo más de la situación clínica rígida de los sistemas de entrenamiento habituales. Consideran que si se desea formar a los terapeutas de conducta adecuadamente para el próximo siglo hay que hacer esto. Este modelo implica que el terapeuta de conducta trabaja en diferentes tiempos con individuos, grupos y sistemas (organizaciones, familias o comunidades). Además, los cambios en el servicio de cuidado de la salud ha dado más importancia a la intervención psicoeducativa y a otras formas de intervención en grupo. Reconocen, igualmente, que en Estados Unidos en los próximos años, el futuro a nivel de empleo estará básicamente en el campo de la psicología de la salud (ver Cummings, 1995). Por ello también será muy importante que sea capaz de predecir los costes de sus tratamientos, un aspecto nuevo hasta hace muy poco sin relevancia. Cada vez se introducen nuevos profesionales en los distintos campos, con menores costes; cada vez se proporcionan servicios básicos a bajo coste; las organizaciones quieren a gente que sea versatil; los cambios sociales son hoy rápidos y extensos. El terapeuta de conducta, como otros profesionales, tienen que adaptarse a ello.

También, los estudiantes de psicología norteamericanos aprecian antes de finalizar sus estudios que lo que se les enseña no tiene que ser necesariamente lo mejor para su práctica (p. ej., Buckley y Turner, 1996). En nuestro país, por el propio sistema de formación, ésta es una queja habitual.

Realmente, lo cierto es que la supervivencia de la terapia de conducta pasa por la transferencia de su tecnología (Strosahl, 1995). Todavía hoy muchos administradores de salud siguen pensando que la terapia de conducta es cara, de ahí que la desechen a favor de otros tratamientos psicológicos. El haberse centrado la evaluación de la terapia de conducta básicamente en la eficacia y haber dejado a un lado el análisis de los costes está en la base de todo ello. Hoy prima el coste-efectividad (Echeburúa, 1998). Un procedimiento para que se implante tiene que ser idealmente eficaz, eficiente y barato o que en el análisis coste-eficiencia salga bien parado. Las palabras de Strosahl (1995) son bien elocuentes cuando dice: "yo pido una moratoria de los artículos que discuten por qué los clínicos entrenados conductualmente no siempre incorporan la investigación a su práctica clínica. Esto es irrelevante para el futuro de la terapia de conducta. Cada uno de nosotros sabemos que los pacientes de la vida real son más complicados que los pacientes de la investigación. Esto es, la investigación es investigación y la práctica clínica no es investigación" (p. 132). 
Por ello, en los programas de formación hay que dar también un peso específico a esta cuestión para que luego los profesionales no se encuentren sin habilidades para hacerle frente a esto. No hay que olvidar tampoco el markéting de las compañías farmacéuticas (Strosahl, 1995), convenciendo a la gente de que la solución está en la píldora o el fármaco, y de que hay un fármaco o una píldora para cualquier padecimiento y que, además, funciona. Esto lleva a que aunque en ocasiones los tratamientos conductuales en algunos trastornos son mucho más efectivos que los fármacos la población cree lo contrario e, incluso, algunos profesionales también lo ignoran por la presión del markéting sobre ciertos fármacos, incluso a veces con publicidad en los propios medios de comunicación o a través de noticias o entrevistas a profesionales $\mathrm{o}$ investigadores relevantes. $\mathrm{La}$ solución pasa tanto por la difusión de nuestros procedimientos efectivos como por tener presencia en los lugares donde se van a tomar decisiones sobre ello o en los lugares de discusión adecuados.

En esta línea, Hayes (1995), en un agudo artículo, cuenta su experiencia personal y sugiere la necesidad de utilizar lenguajes distintos al hablar de los procedimientos conductuales a los estudiantes de psicología, que a los administradores. Mientras que los primeros entienden bien los datos empíricos, los segundos solo saben de eficacia y eficiencia. De ahí que para éstos como para otros lo mejor es hablarle de los métodos conductuales con palabras de sentido común y sin ninguna jerga conductual, y sin abstracciones, ya que no las entenderían.

Por tanto, son necesarias nuevas habilidades para responder al cambio del sistema sanitario, tal como está ocurriendo en todos los países, desde Estados Unidos al nuestro. Dado que los tratamientos conductuales se están adaptando lentamente a esta nueva realidad, es preciso, como indica algún autor (p. ej., Bergan, 1995), que los programas de entrenamiento necesitan una importante adaptación "para producir profesionales que puedan competir en el actual mercado de trabajo" (p. 162). En la consideración de Bergan (1995), es necesario que los psicólogos también tengan conocimientos de economía y costes del servicio, junto a una sólida formación en el campo del tratamiento. Es preciso un cambio en el tratamiento, siendo necesario que los terapeutas de conducta se habitúen a utilizar grupos, por el tema de los costes, y porque la eficacia diferencial respecto al tratamiento individual no es significativa o lo es poco. Sería también necesario una mayor incidencia en programas breves.

Las lecturas de unos y de otros: comparativamente los investigadores en exceso, los clínicos en defecto

Una clara discrepancia de ayer y de hoy entre investigadores y clínicos es lo que leen unos y otros. Mientras que el investigador está dedicado horas y horas del día a la lectura de artículos científicos y libros sobre su temática de estudio, el clínico en raras ocasiones tiene tiempo para ello o, cuando más, no dedicará más que unas pocas horas a leer libros o revistas científicas. Al mismo tiempo, el clinico se encuentra habitualmente que en dichos libros o revistas no ve reflejado su trabajo, en el sentido de las discrepancias que habitualmente hay entre investigación y clínica como ya hemos repasado.

Los investigadores lo que hacen es difundir su información en revistas especializadas, donde predomina un control metodológico riguroso. Pero estas revistas en ocasiones van más dirigidas a los científicos que a los clínicos, porque éstos no las leen y los primeros sí, aunque realmente si no se aplican luego los conocimientos la utilidad de lo descu- 
bierto o comprobado puede ser escasa o nula. Falta, o falla, la "difusión» del descubrimiento.

Como puede verse por ejemplo en Suinn (1993), donde publican los científicos es en revistas que escasamente son leídas por los clínicos. Además, entre lo que conocemos los científicos y lo que hace la gente, como muestra crudamente el estudio de Seligman (1995) del Consumer Report, sobre le eficacia de la psicoterapia tal como la ven los norteamericanos, hay un abismo. Además, los clínicos encuentran poco útil los estudios porque muchos sujetos son excluidos de ellos, lo que les hace ver un abismo entre lo publicado en revistas de alto nivel y su práctica clínica cotidiana. Como resume Sobell (1996) «los científicos publican sus hallazgos de investigación clínica y luego esperan que el «campo» adopte sus intervenciones «exitosas». Si los científicos conductuales estuviesen en una empresa, su sustento seguramente estaría en peligro. Lo que yo y otros científicos no hemos sabido hacer ha sido comercializar nuestros productos para la comunidad de clínicos» (p. 299).

Todo ello ha llevado recientemente a la Association for Advancement of Behavior Therapy, en 1995, a crear una revista específica para reintegrar la ciencia con la práctica de la terapia de conducta, la Cognitive and Behavioral Practice. La idea es que aquellas investigaciones innovadoras, y aquellos artículos con la aplicación de la terapia de conducta al mundo real se publiquen en esta revista. El motivo de ello es que mucha de la literatura científica publicada, incluyendo parte de la conductual, no puede ser aplicada directamente a la práctica clínica (Peterson, 1995). Dado que la práctica clínica de la terapia de conducta se basa en los procedimientos derivados empíricamente, la necesidad de esta revista es clara. También ha aparecido, publicada por Wiley, otra revista de las mismas caracte- rísticas bajo el título de In Session. Su objetivo es mostrarle al clínico el trabajo cotidiano de los terapeutas, aunque en este caso va dirigido a todos los psicólogos clínicos, más que específicamente a los terapeutas cognitivo-conductuales.

En todo lo anterior subyace algo que se sostiene teóricamente pero que luego no se ve siempre reflejado en la práctica: la terapia de conducta se caracteriza (teóricamente) por ir en ella muy unida la investigación y su práctica. Por ello, se espera que la investigación incida en la práctica y que dicha práctica $-\mathrm{y}$ la comunicación pública de sus resultados - también incida en lo que se investiga. Esto no siempre ha sido así, y el que se reconozca ya es un paso importante. Sin embargo, la realidad es que los clínicos leen poco, porque tampoco tienen mucho tiempo, y los investigadores no siempre seleccionan aspectos de un problema que les parezca relevante a los clínicos, o los hallazgos no se comunican bien, o los cauces de comunicación no son todo lo adecuados que debieran ser.

El surgimiento de guías terapéuticas probablemente va a ayudar a que en el futuro se produzca un cambio en este sentido, y el surgimiento de las dos revistas anteriores dedicadas exclusivamente a la clínica son un buen indicio. Con ello se producirá un mayor acercamiento de lo que se investiga a lo que se hace en el "mundo real".

Dado que el tiempo que se le dedica a distintas actividades dentro de la terapia tiene distinto valor, el tiempo disponible debe ser adecuado a ello, para optimizarlo y dedicar el tiempo a actividades que tienen valor real a nivel terapéutico. Por ejemplo, O'Donohue (1995) presenta varias actividades inadecuadas que se llevan a cabo en ocasiones dentro de la terapia, como patologizar al cliente gratuitamente (se infiere lo que tiene antes de hacerle la entrevista y la evaluación); dar consejo sobre qué debe hacer para solu- 
cionar su problema, más que entrenarle en estrategias de solución de problemas; solucionar la crisis de la semana previa del cliente; utilizar instrumentos de evaluación con malas propiedades psicométricas; aplicar terapias sin validación empírica; evaluar factores irrelevantes; $y$, utilizar la terapia para las necesidades personales del terapeuta, solucionar sus problemas o para satisfacer sus intereses.

Por tanto el clínico dedica poco tiempo a la lectura y es necesario que lo que lee sea útil para su práctica. No puede estar varias horas diarias leyendo o no desea hacerlo. El disponer de revistas especializadas, manuales concretos orientados a la clínica y cursos de formación orientados a la práctica son algunas de las soluciones para evitar esta discrepancia que siempre ha estado presente entre la clínica y la investigación.

\section{La primacía de varios problemas} clínicamente representativos y su tratamiento

La prevalencia de los trastornos más importantes son, en primer lugar los trastornos de ansiedad y, en segundo lugar, el abuso de sustancias. En Estados Unidos solo el coste en trastornos de ansiedad representa el $32 \%$ del coste anual de todo el coste en salud mental (Turner, Beidel, Spaulding y Brown, 1995).

Un estudio de gran interés es el de Turner et al. (1995) para conocer los costes de los tratamientos conductuales y cognitivo-conductuales en los trastornos de ansiedad. Utilizando una muestra de 148 miembros del Phobia and Anxiety Disorders Special Interest Group de la AABT, les preguntaron varios aspectos sobre el tipo y coste del tratamiento que aplicaban a seis trastornos de ansiedad: trastorno obsesivo-compulsivo, trastorno de pánico con y sin agorafobia, fobia social, trastorno de ansiedad generalizada, fobia específica y fobia a la sangre/lesión o inyecciones. De los que contestaron, el $55 \%$ trabajaba en la práctica privada, el $21 \%$ en centros universitarios, otro $21 \%$ en la combinación de hospitales privados, práctica privada y agencias de la comunidad y el $2.4 \%$ restante en agencias de la comunidad. De todos ellos el $88 \%$ se consideraban cognitivo-conductuales y el $12 \%$ conductuales.

La duración del tratamiento está mayoritariamente entre 3 y 6 meses, aunque en el caso de la fobia a la sangre/agujas/daño es menor. El método que utilizan más para el trastorno obsesivo compulsivo es la exposición con prevención de la respuesta $(71.4 \%$ de los casos), seguida de ésta combinada con otros procedimientos $(14.3 \%)$ y terapia de conducta cognitiva $(14.3 \%)$. Para el trastorno de pánico, utilizan la terapia de control del pánico $(35 \%)$, terapia cognitivo-conductual $(32.5 \%)$, exposición, terapia de control del pánico, reeducación respiratoria, terapia cognitivo-conductual y biofeedback $(25 \%)$ y solo exposición (7.5\%). Para la fobia social el tratamiento combinado de exposición, técnicas cognitivo-conductuales y entrenamiento en habilidades sociales $(78.8 \%)$, estrategias cognitivoconductuales (15.1\%) y exposición (6.1\%); para el trastorno de ansiedad generalizada básicamente relajación y terapia cognitiva-conductual (82.9) y, en menor grado, tratamiento combinado de biofeedback, medicación y exposición imaginaria $(5.7 \%)$ y relajación $(2.9 \%)$. Para la fobia específica, desensibilización sistemática, imaginaria o en vivo (51.4\%), terapia cognitiva-conductual $(18.9 \%)$ y exposición (8.1\%). Y, para la fobia a la sangre/daño/inyecciones el tratamiento combinado de relajación aplicada, algún tipo de exposición, terapia cognitivo-conductual, relajación, hipnosis y desensibilización sistemática ( $44 \%$ ) junto a terapia cognitiva-conductual (20\%), exposición $(20 \%)$ y relajación aplicada $(16 \%)$. 
A nivel de costes, éstos oscilan en dólares, entre 1.000 y 4.000 . También se utilizan ampliamente sesiones de apoyo, aunque para ellas no les fue posible determinar su coste, por no saber si están o no incluidas en el coste que indica el terapeuta para todo el tratamiento. Un dato interesante que encuentran en este estudio es que cuando se utiliza un tratamiento combinado, éste exige más tiempo $y$, consiguientemente, el coste se incrementa. Apuntan tentativamente que ello puede ser debido a no tener claro qué tratamiento tiene que aplicar el terapeuta.

Para lo que aquí estamos analizano, este estudio es de gran interés porque nos muestra el funcionamiento real de los terapeutas con sus clientes y nos acerca a lo que hacen realmente en la práctica. Lo primero que se aprecia es que el número de sesiones en la clínica es superior al de la investigación. Esto parecería confirmar que lo que encontramos en la clínica son casos más difíciles, más complejos, más deteriorados o, como explicación alternativa, que el clínico prefiere tener más sesiones con el cliente hasta que este solvente totalmente su problema. Notemos que en la investigación, cuando se finaliza el protocolo de sesiones previstas se da de alta al paciente haya o no mejorado. En la clínica no ocurre esto, y ésta es quizás la razón más importante del mayor número de sesiones.

Otro valor de este estudio es que nos presenta los costes reales de los tratamientos. Esta es una cuestión importante, dada la necesidad de conocer cada vez más los costes que tiene cada tratamiento, o la media de coste para un sujeto. Esto permite comparar la eficacia con otros tratamientos, tanto de tipo psicológico como farmacológico.

No debemos tampoco dejar de plantear que no siempre la terapia de conducta se ha interesado por todos los problemas clínicos. Trastornos como la esquizofre- nia no han tenido nunca un interés importante, a pesar de importantes aportaciones; los trastornos de personalidad han sido evitados hasta hace poco; etc. En cambio, la irrupción de las técnicas conductuales en el campo de la salud ha sido muy importante desde los años 70 , al tiempo que se convertía en un área de intervención prioritaria, especialmente debido a las posibilidades profesionales que abría el mismo, surgiendo así la psicología de la salud.

Sobre la eficacia de la intervención clínica en la investigación y práctica clínica

Se parte de la base de que aquello que se obtiene en un estudio de investigación va a ser obtenido de igual modo posteriormente en la clínica. Se asume que los resultados de una investigación clínica son isomórficos a los que obtendrá posteriormente el psicólogo en su consulta. Por lo que hemos visto hasta aqui, esto no tiene porque ser así, dado que no hay tal isomorfismo entre la investigación y la clínica. Sin embargo, siempre se ha sostenido que la eficacia en la clínica era igual o aún mejor que la obtenida en la clínica, dado el tratamiento individualizado, adaptación del tratamiento al cliente, mayor número de sesiones si es necesario, etc. Desde una perspectiva empírica, se ha planteado esta cuestión.

Weisz y colaboradores (p. ej., Weisz, Weiss y Donenberg, 1992; Weisz, Donenberg, Han y Weiss, 1995) han criticado el que las conclusiones que se obtienen en distintos metaanálisis sólo tienen validez en la investigación, sin conocerse qué ocurre realmente en la clínica. Ciertamente, hay pocos estudios en los que se analiza la eficacia de las terapias psicológicas en un contexto clínico más que experimental. Recientemente, Shadish, Matt, Navarro et al. (1997) han reanaliza- 
do 11 metaanálisis previos sobre esta cuestión, llegando a concluir que se obtienen los mismos resultados bajo investigación que en la práctica clínica.

Por tanto, la evidencia disponible, aunque escasa, nos viene a indicar que los resultados que se obtienen en la investigación parecen obtenerse de modo semejante en la clínica. Es de esperar que próximos estudios nos permitan concretar más esta cuestión e, igualmente, conocer cómo mejorar la eficacia de las intervenciones en la clínica, cuando el terapeuta lleva a cabo los cambios que hemos comentado anteriormente (p. ej., adaptar el tratamiento para un específico cliente).

\section{Puntos fuertes y débiles de la terapia de conducta}

Un modo de conocer un poco más las discrepancias que nos encontramos entre investigación y práctica clínica nos viene de la mano de los puntos fuertes y débiles de la terapia de conducta que han presentado Goldfried y Castonguay (1993). Los mismos son relevantes, dado que estos autores propugnan el integracionismo, partiendo de la terapia de conducta. Para ellos, la realidad actual es que la terapia de conducta tiene una gran importancia clínica y que además ha influido de modo directo o indirecto a las otras intervenciones terapéuticas, en el sentido de que la mayoría de los terapeutas norteamericanos se identifican como conductuales o cognitivo-conductuales (Mahoney, 1991) y, al tiempo, otras orientaciones, especialmente la psicodinámica y la experiencial han incorporado técnicas conductuales a sus prácticas, en la línea del eclecticismo o del más reciente movimiento del intregracionismo. Aún así, como afirman, la terapia conductual sigue siendo la principal fuerza de la psicoterapia. Al tiempo, la terapia de conducta siempre ha sido crítica con ella misma y abierta al análisis de sus limitaciones desde sus inicios. En el artículo de Goldfried y Castonguay (1993) hacen esto, presentando 6 puntos fuertes y de éstos sus limitaciones y las nuevas direcciones que se apuntan. Estos puntos fuertes son: 1) la terapia conductual nos proporciona característicamente un análisis detallado de cómo reacciona el individuo ante situaciones específicas de la vida; 2 ) la terapia conductual se ha dedicado normalmente al desarrollo y estudio de técnicas específicas efectivas; 3 ) la terapia conductual hace uso de la orientación de entrenamiento en habilidades; 4) la terapia conductual se centra principalmente en la situación vital presente del cliente; 5) la terapia conductual ha favorecido la investigación de los resultados de la psicoterapia; y, 6) la terapia conductual ha desarrollado diversas formas de intervención para reducir sintomatologías específicas.

Respecto al primer punto fuerte «la terapia conductual nos proporciona característicamente un análisis detallado de cómo reacciona el individuo ante situaciones específicas de la vida", lo que ésta hace es diseccionar los problemas del individuo en variables que puedan ser medidas en términos especificos. Se da más importancia a lo específico que a lo general, y ésta puede ser una limitación en el sentido de que cuanto más específico menos visión de lo global, dejando a un lado el interés por los patrones de conducta, patrones que en ocasiones pueden ser estables y no cambiar de una a otra situación. La nueva dirección que los autores proponen es centrarse no solo en lo específico sino también en los patrones generales de la vida de los clientes, dando importancia a un enfoque que subraye las relaciones interpersonales, teoría de sistemas para la terapia marital conductual, etc.

El segundo punto fuerte "la terapia conductual se ha dedicado normalmente 
al desarrollo y estudio de técnicas específicas efectivas», es indudable la aportación de las técnicas conductuales para el tratamiento de múltiples problemas. Además, las técnicas conductuales están claramente especificadas, se pueden aprender fácilmente, se puede investigar su utilidad y eficacia, y es posible su constante perfeccionamiento. Dejando esto claro los autores indican que en ello puede haber dos limitaciones: las diferencias entre el cliente individual y el terapeuta y el principio subyacente de cambio. Respecto a la primera, parece que el mito de la uniformidad de Kiesler (1966) sería aquí aplicable, dado que en las investigaciones conductuales las diferencias individuales se consideran «error» o "ruido», sin tomarlas en consideración porque se asume que tienen una importancia mínima. Sobre este aspecto los autores apuntan que sí hay diferencias entre específicas diferencias individuales de los clientes (p. ej., locus de control) y el resultado del tratamiento. Lo que sugieren está en la línea del actual "emparejamiento» (matching) sugerido en distintos campos (p. ej., Miller et al., 1995, para el alcoholismo). Esta va a ser una nueva dirección, aunque actualmente hay poca investigación sobre ello.

Sobre la segunda de las limitaciones al segundo punto fuerte, el pasar por alto el principio de cambio, al pensar en términos de técnicas se puede perder de vista el principio que subyace al cambio a través de esa técnica. A este aspecto los autores lo consideran de gran relevancia, dado que en el momento en que se pensase en términos de principios de cambio, ello permitiría considerar otros modos distintos de intervención al cognitivo-conductual, en la línea de su propuesta de la integración (Goldfried, 1992).

El tercer punto fuerte se refiere a que "la terapia conductual hace uso de la orientación de entrenamiento en habili- dades», en el sentido de que se ha adoptado un modelo educativo en vez de un modelo de enfermedad, para enseñar a los clientes habilidades para manejar los problemas de la vida real. Este punto fuerte, en ocasiones puede ser una limitación, al ser excesivamente didáctico y directivo con algunos clientes. Los autores proponen como nueva dirección el constructo de «alianza de trabajo» o clásica "alianza terapéutica», surgido de la tradición psicodinámica. Curiosamente, en el estudio de Raue, Castonguay y Goldfried (1993), encontraron que los terapeutas cognitivo-conductuales valoraban más la alianza terapéutica que los terapeutas psicodinámicos. Este constructo se ha mostrado especialmente útil en trastornos de personalidad, y concretamente en la terapia conductual dialéctica de Linehan (1993), donde uno de sus primeros pasos es establecer con el cliente una relación empática, no directiva y de aceptación. Sólo cuando se logra esto se pasa a la aplicación de las distintas técnicas de intervención.

El cuarto punto fuerte se refiere a que "la terapia conductual se centra principalmente en la situación vital presente del cliente»; esto es, en el aquí y ahora. El pasado tiene poca o ninguna relevancia, y escasas o nulas las experiencias infantiles, elemento básico de la orientación psicodinámica. En cambio, a nivel conductual, nos centramos en el presente, y por ello tiene gran relevancia la asignación de tareas para casa, para que a través de ellas los clientes se adapten a la situación actual. Las limitaciones que apuntan los autores sobre este punto fuerte son dos: los aspectos relativos a lo que ocurre dentro de cada sesión, y las propias reacciones del terapeuta ante el cliente. Sobre la primera, y dado que su objetivo es centrarse en las tareas para casa, se puede perder de vista lo que ocurre dentro de la propia sesión de tratamiento. En ocasiones, en ésta el cliente 
puede presentar demandas que si no se atienden puede limitar, retrasar o hacer fracasar el tratamiento. Por ello, considera que en el futuro habrá que dar más peso a la expresión emocional del cliente, analizar cómo ve y cómo reacciona el cliente ante el terapeuta, prestar especial atención a si no quiere hacer las tareas para casa o las boicotea. Quizás hayan sido Kohlenberg y Tsai (1991) quienes han propuesto, a través de su psicoterapia analítica funcional, quienes más han insistido en este punto, al considerar elemento central del tratamiento una buena interacción terapéutica.

La segunda limitación se refiere a las propias reacciones del terapeuta ante el cliente. Siguiendo el estudio de Henry, Schacht y Strupp (1990) donde han demostrado que los clientes pueden llevar a que el terapeuta muestre hostilidad hacia ellos, la clásica «contratransferencia», el terapeuta conductual no sólo debe centrarse en la aplicación de las técnicas sino también en que esto no ocurra, y ser consciente de que puede ocurrir. Este es un aspecto que puede verse en el futuro como de gran relevancia.

El quinto punto fuerte es que «la terapia conductual ha favorecido la investigación de los resultados de la psicoterapia», y por ello la unión entre investigación y práctica clínica ha sido siempre un elemento definidor de la terapia de conducta. Realmente, la terapia de conducta ha revolucionado el campo de la psicoterapia, en la perspectiva histórica desde su aparición hasta el momento actual. Pero ello, también, tiene en opinión de los autores la limitación de que se ha centrado en la investigación del resultado y ha dejado de lado la investigación del proceso de cambio. Actualmente hay un gran interés en este tema, y se irá acrecentando en el futuro. Aunque hasta ahora se analizaba la cuestión de modo global, el análisis del "proceso terapéutico», hoy hay un mayor interés en analizar algo más específico y más relevante, el "proceso de cambio". Sin embargo, son pocos los terapeutas conductuales interesados por esta cuestión.

El sexto, y último punto fuerte, atañe a que «la terapia conductual ha desarrollado diversas formas de intervención para reducir sintomatologías específicas». Esto ha traído como ventaja que no ha desarrollado un procedimiento de intervención general para todos los problemas, sino técnicas específicas para problemas específicos. La limitación que le achacan a esto es que la terapia de conducta ha prestado especialmente su atención a los problemas del Eje I pero no a los del Eje II, a los trastornos de personalidad, a los que equiparan con la "complejidad de los problemas interpersonales». Nótese que históricamente no se aceptaba ni siquiera el concepto de personalidad, pero la realidad de su existencia y de su gran incidencia hace necesario una intervención en estos complejos trastornos. Y precisamente la intervención conductual en este campo ha retomado conceptos de otras orientaciones e investigaciones no conductuales que han mostrado ser prometedoras (p. ej., Linehan, 1991). De igual modo, Turner (1993) ha propuesto la terapia cognitivo-conductual dinámica, la cual integra la psicología cognitiva, la terapia cognitivo-conductual y la terapia psicodinámica de orientación cognitiva, para ser aplicada a problemas interpersonales de los clientes. Algo semejante ocurre con todo el movimiento constructivista (p. ej., Mahoney, 1991) y con otros terapeutas cognitivo-conductuales (p. ej., Beck y Freeman, 1990).

Goldfried y Castonguay (1993) concluyen que no se puede dudar de la utilidad y eficacia de la terapia cognitivo-conductual, de como otras orientaciones han sido influenciadas por ella, pero que como cualquier otra tiene también sus limitaciones, concluyendo que "hemos defendido (Goldfried, Castonguay y 
Safran, 1992) que el enfoque cognitivoconductual, así como otras orientaciones importantes, seguirán manteniendo sus posiciones predominantes en la psicoterapia del futuro. Aunque la terapia conductual mantendrá probablemente su integridad y contacto con sus raíces, su desarrollo puede verse enriquecido mediante la incorporación de las contribuciones teóricas y clínicas de otras orientaciones» (p. 522).

\section{DE LA TEORÍA A LA PRÁCTICA Y DE LA PRÁCTICA A LA TECNOLOGÍA}

Poco después del surgimiento de la terapia de conducta ha venido coleando una cuestion que hoy permite entender, o es un elemento más, pero importante, para comprender el estado de cosas imperante en la terapia de conducta actual: la relación entre ciencia (o teoría) y tecnología (o aplicación de las técnicas de tratamiento a distintos trastornos). Como dice Rachman (1997), la teoría del aprendizaje jugó un papel claro en la aparición de la terapia de conducta; sin ella hoy no existiría. Pero este papel va decreciendo al decrecer paralelamente el interés en construir teorías. Es el paso de la ciencia a la teconología (Wolpert, 1992). Para Rachman (1997), la introducción de los conceptos cognitivos en terapia de conducta lo que ha conseguido es llenar el vacio entre ciencia y tecnología, lo que él atribuye a la rápida aceptación de esta orientación en pocos años, y la insatisfacción con un empirismo extremo y no siempre conectado con la realidad. Lo cierto es la diversidad siempre ha caracterizado al campo de la terapia de conducta (Beck, 1999).

A principios de los años 70 cobró relevancia la discusión entre ciencia y tecnología, referida a la terapia de conducta, con la introducción del concepto de «ingeniero conductual». La investigación de laboratorio proporcionaba principios y técnicas, desde una perspectiva científica basada en la teoría, pero era necesario dar un paso más: su aplicación al mundo real. Con esa aplicación comenzaban los problemas, como el distinto funcionamiento de esos principios y técnicas del laboratorio a la vida real (Schröeder, 1978). Eso produjo las primeras e importantes insatisfacciones entre la teoría y la práctica que poco a poco fue abriendo fisuras en la terapia de conducta, abriendo la misma hacia otras perspectivas y haciendo su visión más amplia.

La solución que dieron algunos autores ante este hecho fue insistir en que primase la tecnología más que la teoría. Autores como Arthur (1971), Lazarus (1971) y London (1972) son representativos de esta línea, llegando a afirmar que el clínico no necesitaba teorías ni marcos de referencia teóricos, sino técnicas que tenían que funcionar de modo eficaz. Las palabras de London (1972) sobre esta cuestión eran bien representativas: «lo urgente en este momento es crear una práctica sistemática y una tecnología que le dé apoyo: nuevos equipos, nuevas drogas, nuevos aparatos y aditamentos deberán ser la base para ir creando sistemáticamente nuevos métodos de modificación de conducta y para dar dinamismo a las técnicas ya establecidas mediante una comprobación experimental controlada. En lugar de nuevos principios o de justificar los ya gastados, debemos buscar nuevas explicaciones. ¿Cómo tratar tal caso si se tiene tal maquinaria? ¿Qué necesitaría para erigirla? Y, finalmente, para determinar lo que significa ¿qué hacer?» (p. 918). Otro argumento importante que apunta London (1972) es que toda buena tecnología acaba echando por tierra una mala teoría. Por ello sugiere que si una técnica funciona, posteriormente hay que comprobar para quién y cuándo se puede aplicar. 
Comprobar cómo y por qué funciona vendría después, y sería claramente menos relevante que lo anterior. Estamos ante el pragmatismo clínico, tantas veces reñido con partir de la teoría y forzar la práctica en función de ella, tan característico a la psicología.

Fue Arthur (1971) uno de los autores que más abiertamente ha defendido un enfoque de "ingeniería conductual», término que se ha mantenido hasta el presente dentro del análisis conductual aplicado. Arthur (1971) distinguía entre la ciencia teórica, la ciencia empírica y la ciencia ingenieril. Posteriormente, Yates (1975), resumía el estado de la cuestión, indicando las razones por las que los aspectos esenciales de la investigación científica, desde un marco teórico que no clínico, se convertía en inadecuada para la toma de decisiones clínicas: 1) un estudio teórico no evalúa las posibilidades prácticas de los resultados obtenidos; 2 ) un estudio de laboratorio no tiene en cuenta los efectos moderadores de las variables existentes en la vida real; 3 ) los conceptos teóricos que se emplean (por p. ej., ansiedad) en un estudio de laboratorio pueden tener poca relación con la situación en los pacientes reales (p. ej., los pacientes con ansiedad real); 4) los resultados de un estudio teórico no siempre son útiles para pasarlos a la práctica; $y, 5)$ un estudio teórico no demuestra que sus hallazgos sean superiores a los enfoques alternativos. Por contra, un enfoque en el que la investigación se orienta a la acción tiene más probabilidad de dar respuesta a un problema real y permite más facilmente conocer si es útil para solucionarlo.

Esta dicotomía, que surge mismo en los comienzos de la terapia de conducta, tiene quizás a su caso más extremo en Lazarus, quién partiendo de esta dicotomía teoría-práctica, optó desde muy pronto por una primacía de la práctica en contra de la teoría, lo que le llevó a for- mular a principios de los años 70 su conocido eclecticismo técnico y su terapia de conducta multimodal (Lazarus, 1971, 1976), posteriormente redenominada como terapia multimodal (Lazarus, 1981), la cual con el transcurrir de los años encuentra un buen lugar de acomodo en los movimientos de la integración de la psicoterapia (p. ej., Lazarus, 1997), y en donde el papel de Lazarus ha sido importante por su quehacer real hacia este movimiento.

El hilo conductor del planteamiento de Lazarus es que cualquier procedimiento científico basado en la experimentación de cualquier terapeuta de cualquier enfoque que sea útil, es aplicable, aparte del modelo teórico del que haya partido. Este enfoque lleva a que al final se utilice cualquier técnica o terapia que "funcione». Esto llevó a Lazarus a plantear su perfil multimodal o BASIC ID. El BASIC ID son las iniciales de las áreas problema que el clínico debe investigar en su cliente, y que toman su nombre de la primera palabra de las siete áreas que se deben evaluar, que en inglés son behavior (B, conducta), affect (A, afecto), sensation (S, sensaciones), imagery (I, imágenes), cognition (C, cognición), interpersonal relations (I, relaciones interpersonales), y drugs ( $D$, drogas y fámacos), aunque en este caso se refiere a factores biológicos, pero aplica la palabra drogas para tener el acrónimo BASIC ID, por las connotaciones que ello tiene, más que BASIC IB, que no tiene ese mismo significado. Partiendo de estas siete áreas, se aplican técnicas específicas para aquellos aspectos deficitarios del sujeto en ellas.

Como nota de interés histórico, debemos a Arnold A. Lazarus ser la primera persona, según muchos, que acuñó por primera vez el término terapia de conducta en 1958 (Lazarus, 1958), y también precozmente uno de los autores que produjo una ruptura con los principios ini- 
ciales de la terapia de conducta basada en el aprendizaje.

Encasillar a Lazarus siempre ha sido un problema dentro de la terapia de conducta, aunque recientemente da la sensación que ha vuelto, y con su perspectiva, a la misma al hacerse la terapia de conducta también más abierta (p. ej., Lazarus, 1997). Por ejemplo, son bien representativas las valoraciones de Wilson (1982) cuando afirma que 30 de las 39 terapias que Lazarus incluye en su terapia multimodal son técnicas habituales de la terapia de conducta, aunque también le criticaron que su modelo de tratamiento no especifica claramente qué técnicas utilizar en un caso concreto, dejando una total libertad al clínico.

Como resumen de todo lo anterior, la investigación siempre parte de un modelo teórico, que en el caso de la terapia y modificación de conducta fue inicialmente la psicología del aprendizaje, y posteriormente toda la psicología científica, facilitando con ello el descubrimiento de tratamientos eficaces que fueron aplicados a distintos trastornos clínicos. Una vez que ha quedado demostrado que los mismos son eficaces, ya tenemos una tecnología de cambio eficaz, lo que acarrea como consecuencia la aplicación de la misma a nuevos problemas, para ampliar su campo de intervención. $\mathrm{Al}$ incrementarse la demanda social por su eficacia, va pasando a convertirse cada vez más en "tecnología», como ha sido históricamente facilitada por la aceptación social de la psicología y su implantación como una ciencia "respetable» en el contexto social, y con una "estructura organizativa», operativa y eficiente en su introducción y expansión social. Finalmente, la primacía de la tecnología ya viene no solo del medio social sino también de sus propios aplicadores, los clínicos en este caso, los cuales tienen que dar respuesta a problemas cotidianos, importándoles menos o poco las bases empíricas y teóricas de lo que aplican. Se pasa a primar el pragmatismo social. Un reflejo se ve en la investigación, que depende del dinero para poder investigar y de la priorización de lo que se debe investigar. Por ello el dinero para investigaciones básicas suele ser escaso, a menos que se vea claro su inmediato o posterior traslado de esa investigación para nuevos campos prácticos que se consideren relevantes (p. ej., SIDA). Además, vivimos en un momento de entronización de la tecnología. Un buen ejemplo lo tenemos en la medicina, con un claro predominio de la tecnología, aunque en la psicología todavía en ocasiones hay un predominio de la teoría por delante de las técnicas de intervención y de la tecnología que comportan. Hoy, más que nunca lo importante es demostrar que se es útil y efectivo, tanto ante nuestros colegas como ante el medio social. Y, si ello es así, lo que hacemos se convierte en importante.

\section{DISCUSIÓN Y CONCLUSIONES}

Las discrepancias que nosotros considerábamos que existían en 1986 entre la teoría e investigación y la práctica clínica de la terapia de conducta eran doce (Becoña, 1986), como hemos visto. Aunque en los últimos años se han solventado varias de estas discrepancias (p. ej., diagnóstico psiquiátrico), también han ido surgido otras nuevas (p. ej., la escasa incidencia de la investigación en la práctica clínica). Junto a ello hemos analizado la relación entre teoría y práctica y práctica y tecnología. Esta es, en nuestra consideración, uno de los elementos que explican estas discrepancias, dado que el clínico tiene que resolver problemas cotidianos y tiene que hacerlo efectivamente. De ahí que va adaptando lo que ha aprendido en sus estudios o de los datos que le proporcionan los estudios empíri- 
cos, cuando acude a ellos, para afrontar sus dificultades cotidianas cuando no tiene a su alcance un modo efectivo de abordarla. De ahí, que pensamos que esta dicotomía se va a seguir manteniendo.

Wolfe (1994) hace notar la diferencia entre las personas que trata y la de sus grupos de investigación. Clement (1996) plantea el problema de integrar la investigación con la práctica. Por ejemplo, indica como los que hacen investigación no suelen hacer práctica privada y, si la hacen, ésta es muy limitada o le dedican poco tiempo a la misma (Moldawsky, 1992). Y, si la hacen, suele estar integrada dentro de lo que es su programa de investigación clínico. Por contra los psicólogos clínicos pueden estar más interesados en recibir nuevos clientes, retenerlos tanto como sea posible, estar a gusto con ellos y obtener ingresos de ellos mediante el pago de las sesiones. Sin embargo, en los estudios controlados que demuestran una relación entre la integración de la investigación y los métodos clínicos nunca se detienen en los anteriores aspectos (p. ej., el del pago).

Fensterheim y Raw (1996) afirman que la investigación de la psicoterapia no es la práctica de la psicoterapia, siendo dos campos distintos. Cada uno de ellos tiene sus propios objetivos, su propio estilo de pensar y sus propios problemas y procedimientos. Notemos, por ejemplo, como el estudio de casos es una fuente potencial de nuevas hipótesis para la investigación experimental, aunque luego en la práctica pocas veces lo es realmente. Creemos, sin embargo, que la introdución del DSM-III a partir de 1980 y sus versiones posteriores, han permitido un cambio que hoy se considera bueno tanto para el investigador como para el clínico. Al menos ahora tenemos un mayor nivel de consenso sobre lo que hablamos. De igual modo, las guías clínicas van a favorecer un mayor diálogo entre clínicos e investigadores, como realmente ya está ocurriendo así en estos últimos años, de ahí el que muchos investigadores y clinicos hayan salido a la palestra para discutir estos temas ( $p$. ej., Hayes et al., 1995).

Sin embargo, mientras que el clínico se diferencia claramente, en lo anterior, del investigador, hay un importante punto de coincidencia entre ambos. Esta coincidencia es en la evaluación. Para Clement (1996) éste puede ser un punto de unión entre la investigación y la práctica clínica. La importancia de que los clínicos presenten series de casos es en este contexto muy importante, para que conozcamos como funcionan realmente los tratamientos. Este es uno de los procedimientos sugeridos por el Task Force on Promotion and Dissemination on Psychological Procedures (1993) para demostrar la eficacia de un tratamiento dado, como veremos en la siguiente parte. Al mismo tiempo es cierto que «el investigador no escribe para el clínico; el investigador escribe para otros investigadores. Los problemas no son aquellos problemas con los que se enfrenta el trabajador clínico sino más bien los problemas de otros investigadores" (Fensterhein, 1993, p. 149). Aunque, por suerte, esto va paulatinamente cambiando, todavía este hecho se sigue apreciando en los artículos publicados en las revistas científicas. Además, como indica Hayes (1996) en un artículo titulado «creando el clínico empírico», muchas de las metodologías de investigación a las que se dedica mucho tiempo de entrenamiento luego no es posible implementarlas en la práctica privada. Esto es negativo e impráctico. Al tiempo, no hay que perder de vista los cambios que se están produciendo en el sistema de salud, que exigen técnicas eficaces, innovadoras y baratas. De no hacer esto, el psicólogo no podrá sobrevivir, de ahí la insistencia permamente de que tengamos tratamientos eficaces y al mismo tiempo baratos (Hayes 
et al., 1995). Y, además, como nos es característico, y como bien dice Nezu (1996) «desafortunadamente, mientras nosotros continuamos ocupados en una guerra civil otros profesionales están más que contentos en proporcionar los servicios de tratamiento en lugar de hacerlo los psicólogos» (p. 162). Por suerte, en los últimos años, ha cobrado gran relevancia el análisis de las diferencias entre los clínicos y los investigadores, esperándose que la apertura de un diálogo entre unos y otros permita solventar algunas de las que anteriormente hemos comentado. Mientras que tenemos mucha información sobre lo que se hace en la investigación, sabemos poco o muy poco de lo que ocurre en la clínica realmente. Conocer lo que ocurre es por ello necesario para que si hay elementos que se pueden mejorar se pueda hacer.

Como un ejemplo de la necesidad de este cambio, en el libro editado por Hayes et al. (1995) pueden verse los distintos puntos de vistos entre teoría y práctica bajo el esquema de la necesidad de desarrrollar estándares adecuados de investigación. Dawes (1995), por ejemplo, insiste en que tiene que haber unos estándares para la práctica y que éstos son los que la deben luego guiar. Davison y Lazarus (1995) proponen el desarrollo del clínico con mente científica, donde combinaría sus cualidades de clínico y científico, como no parece que ello esté ocurriendo hoy. Igualmente dan gran valor al estudio de casos. En el contrapunto, otros autores, como por ejemplo Persons (1995) o Wilson (1995) se plantean las causas por las que los psicólogos clínicos no adoptan aquellos tratamientos que se ha demostrado que tienen una buena demostración empírica de su eficacia. Creemos que la educación continua podría ser una solución a este tema, aunque por el ejemplo norteamericano, donde allí tiene gran relevancia, tampoco lo han resuelto totalmente.
Como consuelo, lo que le ocurre a la terapia de conducta no le es exclusiva de ella, ni exclusiva de la psicología clínica, si hacemos un análisis de lo que ocurre en otras ciencias. Más bien lo que hemos analizado respecto a la discrepancias investigación-práctica clínica en terapia de conducta, parece ser un fenómeno habitual en el campo de la salud mental e incluso a veces de las intervenciones en salud física y en otras ciencias. Como un ejemplo de esto, son bien representativas las palabras de Cees Goos, el Coordinador de la Unidad de Alcohol, Drogas y Tabaco, de la Oficina Regional para Europa de la Organización Mundial de la Salud, el cual haciendo la presentación del documento "Directrices para la evaluación del tratamiento de la dependencia alcohólica», elaborado por la The Plinius Maior Society (1995), un sociedad formada por psiquiatras, representando a importantes psiquiatras, Catedráticos de Psiquiatría y Directores Psiquiatras de Unidades de Tratamiento del Alcoholismo de toda Europa, afirma que: "Con este informe, la Plinius Maior Society ha emprendido un ejercicio "cartográfico" realmente necesario. Si bien durante los últimos 15 años se han conseguido importantes logros en el desarrollo de instrumentos y criterios de diagnóstico sobre el abuso de sustancias, no parece haberse creado una red sistemática de comunicaciones entre los grupos de investigación interesados en este sector. Quizás intervenga aquí, una vez más, la bien conocida disociación entre la investigación y la práctica clínica. Al clínico medio puede parecerle que organismos y grupos diversos han ido estableciendo sus indicadores con lenguajes e incluso orientaciones distintas. Se trataba, pues, de un ejercicio altamente necesario, ya que el tema objeto de este informe es de crucial importancia para diseñar las aportaciones de los profesionales sanitarios» (p. 11). 
Con todo, la necesidad de un mayor acercamiento entre la investigación y la clínica no sólo es necesario sino que es fructífero y útil para ambas. Mas aún, si creemos que el cambio de conducta es posible, debemos nosotros mismos dar los primeros cambios en nuestra propia conducta sobre este aspecto.

\section{REFERENCIAS BIBLIOGRÁFICAS}

American Psychiatric Association (1980). Diagnostic and statistical manual of mental disorders, 3rd ed. Washington, DC: Author (trad. cast.: Barcelona: Toray, 1984)

American Psychiatric Association (1994). Diagnostic and statistical manual of mental disorders, 4th ed. Washington, DC: Author (trad. cast.: Barcelona: Toray, 1994)

American Psychiatric Association (1996). Practice guideline for the treatment of patients with nicotine dependence. American Journal of Psychiatry, 153, Supp. 10, 1-31.

Arnau, J. (1984). Diseños experimentales en psicología y educación, vol. 2. México: Trillas.

Arndorfer, R.E., Allen, K.D. y Aljazireh, L. (1999). Behavioral health needs in pediatric medicine and the acceptability of behavioral solutions: Implications for behavioral psychologists. Behavior Therapy, 30, 137-148.

Arthur, A.Z. (1971). Psychology as engineering and technology of behavior. Canadian Psychologist, 12, 30-36.

Barlow, D.H. (1981). On the relation of clinica research to clinical practice: Current issues, new directions. Journal of consulting and Cliniical Psychology, 49, 147-155.

Barlow, D.H. (1994). Psychological interventions in the era of managed competition. Clinical Psychology: Science and Practice, 1, 109-123.

Barlow, D.H., Hayes, S.C. y Nelson, R.O. (1984). The scientist practitiones. Research and accountability in clinical and educational setting. Nueva York: Pergamon.

Barlow, D.H. y Hersen, M. (1984). Single case experimental designs: Strategies for stud- ying behavior change, 2nd ed. Nueva York: Pergamon (trad. cast., Barcelona: Martínez Roca, 1988).

Bayés, R. (1984). ¿Por qué funcionan las terapias comportamentales? Anuario de Psicología, 30-31, 127-147

Beck, A.T. y Freeman, A. (1990). Cognitive therapy of personality dissorders. Nueva York: Guilford Press (trad. cast.: Barcelona, Paidós, 1995).

Beck, J.G. (1999). Turning 30: Grown-up at last. Behavior Therapy, 30, 1-4.

Becoña, E. (1986). Discrepancias entre la investigación y la práctica clínica de la terapia de conducta. Revista Española de Terapia del Comportamiento, 4, 221-258.

Becoña, E. (1991). ¿Es la modificación de conducta cognitiva más eficaz que la clásica modificación de conducta? Revista de Psicología General y Aplicada, 44, 339-348.

Becoña, E., Lista, M.J. y Froján, M.J. (1988). JPor qué los sujetos recáen en los tratamientos para dejar de fumar. Revista Española de Drogodependencias, 14, 29-36.

Bellack, A.S. y Hersen, M. (1985). General considerations. En A.S. Bellack y M. Hersen (Eds.), Handbook of clinical behavior therapy (pp. 1-19). Nueva York: Plenum.

Bergan, J. (1995). Behavioral training and the new mental health: Are we learning what we need to know. The Behavior Therapist, 18, 161-164.

Bergin, W.E. y Garfield, S.L. (Eds.) (1994). Handbook of psychotherapy and behavior change. Nueva York: Plenum.

Black, M.J. y Schroeder, H.E. (1985). Social inadequacy. En M. Hersen y C.G. Last (Eds.), Behavior therapy casebook (pp. 104-117). Nueva York: Springer.

Blanco, A. (1988). Introducción histórica y conceptual al diagnóstico clínico. En A. Blanco (Ed.), Apuntes de diagnóstico clinico (pp. 7-43). Valencia: Promolibro.

Bootzin, R.R. y McKhight, K.K. (1998). The role of biased information processing in depression: Evaluation and implications for treatment. Behavior Therapy, 29, 619630.

Buckley, T.C. y Turner, S.M. (1996). Bridging the science-practice gap in the 21st century: A student perspective. The Behavior Therapist, 19, 107-108. 
Cateutelli, J.D., Rosenwasser, B.J. y Skinner, L. (1996). A tragic view of behavioral technology and dissemination: Framing the debate for the 21st century. The Behavior Therapist, 20, 165-169.

Clement, P.W. (1996). Evaluation in private practice. Clinical Psychology. Science and Practice, 3, 146-159.

Cone, J.D., Alexander, K., Lichtszajn, T. y Mason, R.L. (1996). Reengineering clinical training curricula to meet challenges beyond the year 2000. The Behavior Therapist, 19, 65-70.

Cummings, N.A. (1995). Impact of managed care on employment and training: A primer for survival. Professional Psychology: Research and Practice, 26, 10-15.

Davison, G.D. y Lazarus, A.A. (1995). The dialectics of science and practice. En S.C. Hayes, V.M. Follette, R.M. Dawes y K.E. Grady (Eds.), Scientific standards of psychological practice. Issues and recommendations (pp. 95-120). Reno, NV: Context Press. Dawes, R.M. (1995). Standards of practice. En S.C. Hayes, V.M. Follette, R.M. Dawes y K.E. Grady (Eds.), Scientific standards of psychological practice. Issues and recommendations (pp. 31-47). Reno, NV: Context Press.

Eadington, E.S. (1984). Statistics and single case analysis. En M. Hersen, R.M. Eisler y R.M. Miller (Eds.), Progress in behavior modification (vol. 16, pp. 83-119). Nueva York: Academic Press.

Echeburúa, E. (1998). ¿Qué terapias psicológicas son eficaces? Un reto ante el 2000. Revista de Psicopatología y Psicología Clínica, 3, 149-160.

Elkin, I. (1994). The NIHM treatment of Depression Collaborative Research Program. Where we began and where we are. En W.E. Bergin y S.L. Garfield (Eds.), Handbook of psychotherapy and behavior change (4th ed., pp. 114-139). Nueva York: Plenum.

Emmelkamp, P.M.G. y Foa, E.B. (1983). Failures are a challenge. En E.B. Foa y P.M.G. Emmelkamp (Eds.), Failures in behavior therapy (pp. 1-9). Nueva York: Wiley.

Eysenck, H.J. (1952). The effects of psychotherapy: An evaluation. Journal of Counseling Psychology, 16, 319-324.
Fensterheim, H. (1993). Letter to the editor. The Behavior Therapist, 16, 149.

Fensterheim, H. y Raw, S.D. (1996). Psychotherapy research is not psychotherapy practice. Clinical Psychology. Science and Practice, 3, 168-171.

Fernández-Ballesteros, R. (1981). Comparaciones entre la evaluación tradicional y la evaluación conductual. En R. FernándezBallesteros y J.A.I. Carrobles (Eds.), Evaluación conductual (pp. 31-62). Madrid: Pirámide.

Fishman, S.T. y Lubetkin, B.S. (1983). Office practice of behavior therapy. En M. Hersen (Ed.), Outpatient behavior therapy. A clinical guide. Nueva York: Grune and Stratton.

Foa, E.B. y Emmelkamp, P.M.G. (Eds) (1983). Failures in behavior. Nueva York: Wiley.

Follette, W.C. (1998). Expanding the domains of clinical behaviorism: A comment on Dougher. Behavior Therapy, 29, 593-601.

Frances, A., First, M.B. y Pincus, H.A. (1997). DSM-IV. Guía de uso. Barcelona: Masson (original, 1995).

Franks, C.M. (1993). Archives. The Behavior Therapist, 16, 133.

Garfield, S.L. (1996). Some problems associated with "validated" forms of psychotherapy. Clinical Psychology: Science and Practice, 3, 218-229.

Goldfried, M.R. (Ed.) (1982). Converging themes in psychotherapy: Trends in psychodynamic, humanistic and behavioral practice. Nueva York: Springer.

Goldfried, M. (1983). The behavior therapist in clinical practice. The Behavior Thrapist, 6, 45-46.

Goldfried, M.R. y Castonguay, L.G. (1993). Behavior therapy: Redefining strengths and limitations. Behavior Therapy, 24, 505-526.

Goldfried, M.R., Castonguay, L.G. y Safran, J.D. (1992). Core issues and future directions in pshchotherapy integration. En J.C. Norcross y M.R. Goldfried (Eds.), Handbook of psychotherapy integration (pp. 593616). Nueva York: Basic Books.

Goldfried, M.R. y Castonguay, L.G. (1993). Behavior therapy: Redefining strengths and limitations. Behavior Therapy, 24, 505-526.

Hayes, S.c. (1981). Single case experimental design and empirical clinical practice. 
Journal of Consulting and Clinical Psychology, 49, 193-202.

Hayes, S.C. (1995). Working with managed care: Lessons from the acceptance and committment therapy training project. The Behavior Therapist, 18, 184-186.

Hayes, S.C. (1996). Creating the empirical clinician. Clinical Psychology: Science and Practice, 3, 179-181.

Hayes, S.C., Follette, V.M., Dawes, R.M. y Grady, K.E. (Eds.) (1995). Scientific standards of psychological practice. Issues and recommendations. Reno, NV: Context Press.

Henry, W.P., Schacht, T.E. y Strupp, H.H. (1990). Patient and therapist introject, intepersonal process and differential psychotherapy outcome. Journal of Consulting and Clinical Psychology, 58, 768-7744.

Hersen, M. (1992). Psychiatric diagnosis and behavioural assessment. En J. Cottraux, P. Legeron y E. Mollard (Eds.), Which psychotherapies in year 2000? (pp. 135-148). Amsterdam: Swets \& Zeitlinger.

Hersen, M. y Last, C.G. (Eds.) (1985). Behavior therapy casebook. Nueva York: Springer.

Howard, K.I. (1998). Some relationships between basic and applied research. Behavior Therapy, 29, 715-719.

Kanfer, F.H. y Saslow, G. (1965). Behavioral analysis. Archives of General Psychiatry, 12, 529-538.

Kanfer, F.H. y Saslow,G. (1969). Behavioral diagnosis. En C.M. Franks (Ed.), Behavior therapy: Appraisal and status. Nueva York: McGraw-Hill.

Kaplan, S.J. (1986). The private practice of behavior therapy. A guide for behavioral practitioners. Nueva York: Plenum.

Kazdin, A.E. (1982). Single-case research design: Methods for clinical and applied settings. Oxford, RU: Oxford University Press.

Kazdin, A.E. (1992). Research design in clinical psychology, 2nd ed. Needham Heights, MA: Allyn \& Bacon.

Kazdin, A.E. (1994). Behavior modification in applied settings, 5th ed. Beldmont, CA: Wastworth, Inc. (trad. cast., México: El Manual Moderno, 1996)

Kiesler, D.J. (1966). Some myths of psychotherapy research and the search for a paradigm. Psychological Bulletin, 65, 110-136.
Kohlenberg, R.J. y Tsai, M. (1991). Functional analytic psychotherapy. Nueva York: Plenum Press.

Labrador, F.J., Cruzado, J.A. y Muñoz, M. (Eds.) (1993). Manual de técnicas de modificación y terapia de conducta. Madrid: Pirámide.

Last, C.G. y Hersen, M. (1985). Clinical practice of behavior therapy. En M. Hersen y C.G. Last (Eds.), Behavior therapy casebook (pp. 3-15). Nueva York: Springer.

Lazarus, A.A. (1971). Behavior therapy and beyond. Nueva York: McGraw-Hill (trad. cast.: Buenos Aires, Paidós, 1980).

Lazarus, A.A. (1976). Multimodal behavior therapy. Nueva York: Springer.

Lazarus, A.A. (1977). Has behavior therapy outlived its usefulness? American Psychologist, 32, 550-554.

Lazarus, A.A. (1981). The practice of multimodal therapy. Nueva York: McGraw-Hill.

Lazarus, A.A. (1994). How certain boundaries an ethics disminish therapeutic effectiveness. Ethics \& Behavior, 4, 255-261.

Lazarus, A.A. (1997). Disenchantment and hope: Will we ever occupy center stage. Behavior Therapy, 28, 363-370.

Linehan, M.M. (1993). Cognitive-behavioral treatment of borderline personality disorders. Nueva York: Guildford Press.

London, P. (1972). The end of ideology in behavior therapy. American Psychologist, 27, 913-920.

Llavona, L. (1984). El proceso de evaluación conductual. En J. Mayor y F.J. Labrador (Eds.), Manual de modificación de conducta (pp. 93-122). Madrid: Alhambra.

Macià, D. y Méndez, X. (Eds.) (1988). Aplicaciones clínicas de la evaluación y modificación de conducta. Análisis de casos. Madrid: Pirámide.

Mahoney, M. J. (1991). Human change processes. Theoretical bases for psychotherapy. Nueva York: Basic Books.

Mandler, G. (1995). The situation of psychology: Landmarks and choicepoints. American Journal of Psychology, 109, 1-35.

Marcus, M.D. (1985). Obesity. En M. Hersen y C.G. Last (Eds.), Behavior therapy casebook (pp. 144-157). Nueva York: Springer.

Marlatt, G.A. y Gordon, J.R. (1985). Relapse prevention. Maintenance strategies in the 
treatment of addictive behaviors. Nueva York: Guildford.

McCrady, B.S. (1986). Implications for behavior therapy on the changing alcoholism health care delivery system. The Behavior Therapist, 9, 171-174.

Miller, W.R. (1987). Behavioral alcohol treatment research advances: Barriess to utilization. Advances in Behaviour Research and Therapy, 9, 145-167.

Miller, W.R., Brown, J.M., Simpson, T.L., Handmaker, N.S., Bien, t.H., Luckie, L.F., Montgomery, H.A., Hester, K.S. y Tonigan, J.S. (1995). What works? A methodological analysis of the alcohol treatment outcome literature. En R.K. Hester y W.R. Miller (Eds.), Handbook of alcohol treatment approaches. Effective alternatives (2nd ed., pp. 12-44). Boston, MA: Allyn and Bacon.

Moldawsky, S. (1992). A proper education for private practice. Psychotherapy in Private Practice, 11, 37-42.

Nelson, R.M. (1983). Behavioral assessment: Past, present and future. Behavioral Assessment, 5, 195-207.

Nezu, A. (1996). What are we doing to our patients and should we care if anyone else knows? Clinical Psychology. Science and Practice, 3, 160-163.

O'Donohue, W. (1995). Time allocation in psychotherapy. The Behavior Therapist, 18, 117-119.

Páez, D., Echeburúa, E. y Borda, M. (1993). Evaluación de la eficacia de los tratamientos psicológicos: una propuesta metodológica. Revista de Psicología General y Aplicada, 46, 187-198.

Persons, J.B. (1995). Why practicing psychologists are slow to adopt empirically validated treatments. En S.C. Hayes, V.M. Follette, R.M. Dawes y K.E. Grady (Eds.), Scientific standards of psychological practice. Issues and recommendations (pp. 141-161). Reno, NV: Context Press.

Peterson, L. (1995). Twin visions: The iterative relationship between our practice and research journals. The Behavior Therapist, 18, 71-73.

Piotrowski, C. y Keller, J.W. (1984). Attitudes toward clinical assessment by members of the AABT. Psychological Reports, 55, 831838.
Rachman, S. (1997). The evolution of cognitive behavior therapy. En D.M. Clark y C. G. Fairburn (Eds.), Science and practice of cognitive behavior therapy (pp. 1-26). Oxford, RU: Oxford University Press.

Raue, P.J., Castonguay, L.G. y Goldfried, M.R. (1993). The working alliance: A comparison of two therapies. Psychotherapy Research, 3, 197-207.

Rogers, E.M. (1995). Lessons for guidelines from the diffussion of innovations. Joint Commission Journal of Quality Improvements, 21, 324-328.

Seligman, M.E.P. (1995). The effectiveness of psychotherapy: The Consumer Reports Study. American Psychologist, 50, 965-974.

Shadish, W.R., Matt, G.E., Navarro, A.M., Siegle, G., Crits-Christoph, P., Hazelrigg, M.D., Jorm, A.F., Lyons, L.C., Nietzel, M.T., Prout, H.T., Robinson, L., Smith, M.L., Svartberg, M. y Weiss, B. (1997). Evidence that therapy works in clinically representative condicions. Journal of Consulting and Clinical Psychology, 65, 355-365.

Skinner, B.F. (1957). Verbal behavior. Nueva York: Appleton-Century-Croft (trad. cast., México: Trillas, 1981).

Sobell, L.C. (1996). Bridging the gap betwen scientists and practitioners: The challenge before us. Behavior Therapy, 27, 297-320.

Strosahl, K. (1995). Behavior therapy 2000: A perilous journey. The Behavior Therapist, 18, 130-133.

Suinn, R.M. (1993). Practice: what should we preach? The Behavior Therapist, 16, 151-152.

Swan, G.E. y MacDonald, M.L. (1978). Behavior therapy in practice: A national survey. Behavior Therapy, 9, 799-807.

Task Force on Promotion and Dissemination of Psychological Procedures (1993). Task force on promotions and dissemination of psychological procedures. A Reports adopted by the APA Division 12 Board, October 1993, 1-17

Taylor, S. (1999). Behavioral assessment: Review an prospect. Behaviour Research and Therapy, 37, 475-482.

The Plinius Maior Society (1995). Directrices para la evaluación del tratamiento de la dependencia alcoholica. Barcelona: Ajuntament de Barcelona, Institut Municipal de la Salut Pública. 
Turner, R. M. (1993). Dynamic cognitivebehavior therapy. En T. Giles (Ed.), Handbook of effective psychotherapy (pp. 437454). Nueva York: Plenum.

Turner, S.M. y Ascher, L.M. (1985). Behavior therapy outcome research: Some methodological issues. En S.M. Turner y L.M. Ascher (Eds.), Evaluating behavior therapy outcome (pp. 1-14). Nueva York: Springer.

Turner, S.M., Beidel, D.C., Spaulding, S.A. y Brown, J.M. (1995). The practice of behavior therapy: A national survey of cost and methods. The Behavior Therapist, 18, 1-4.

Van Hasselt, V.B. y Hersen, M. (Eds.) (1996). Sourcebook of psychological treatment manuals for adult disorders. Nueva York: Plenum.

Yates, A. (1975). Theory and practice in behavior therapy. Nueva York: Wiley (trad. cast.: México, Trillas, 1977)

Weisz, J.R., Donenberg, G.R., Han, S.S. y Weiss, B. (1995). Bridging the gap between laboratory and clinic in child and adolescent psychotherapy. Journal of Consulting and Clinical Psychology, 63, 688-701.

Weisz, J.R., Weiss, B. y Donenberg, G.R. (1992). The lab versus the clinic: Effects of child and adolescent psychotherapy. American Psychologist, 47, 1578-1585.

Wilson, G.T. (1982). Clinical issues and strategies in the practice of behavior therapy. En C.M. Franks, G.T. Wilson, P.C. Kendall y K.D. Brownell (Eds.), Annual review of behavior therapy (Vol. 9, pp. 309-343). Nueva York: Guildford Press.

Wilson, G.T. (1995). Empirically validated treatments as a basis for clinical practice: Problems and prospects. En S.C. Hayes, V.M. Follette, R.M. Dawes y K.E. Grady (Eds.), Scientific standards of psychological practice. Issues and recommendations (pp. 163-196). Reno, NV: Context Press.

Wolfe, B.E. (1994). Adapting psychotherapy outcome research to clinical reality. Journal of Psychotherapy Integration, 4, 160-166.

Wolpe, J. (1958). Psychotherapy by reciprocal inhibition. Stanford: Stanford University Press (trad. cast., Bilbao: Desclée de Brouwer, 1978).

Wolpert, L. (1992). The unnatural nature of science. Cambridge, MA: Harvard University Press.

Zeiss, A. (1997). President's message. The Behavior Therapist, 20, 120. 